\title{
Stellar systems in the direction of Pegasus I
}

\section{Low surface brightness galaxies}

\author{
Nélida M. González ${ }^{1,2}$, Analía V. Smith Castelli ${ }^{1,2,3}$, Favio R. Faifer ${ }^{1,2,3}$, \\ Carlos G. Escudero ${ }^{1,2}$, and Sergio A. Cellone $e^{1,3,4}$ \\ 1 Facultad de Ciencias Astronómicas y Geofísicas, Universidad Nacional de La Plata, Paseo del Bosque, \\ B1900FWA La Plata, Argentina \\ e-mail: ngonzalez@fcaglp.unlp.edu.ar \\ 2 Instituto de Astrofísica de La Plata (UNLP-CONICET), Paseo del Bosque, B1900FWA La Plata, Argentina \\ 3 Consejo Nacional de Investigaciones Científicas y Técnicas, Godoy Cruz 2290, C1425FQB CABA, Argentina \\ ${ }^{4}$ Complejo Astronómico El Leoncito (CONICET - UNLP - UNC - UNSJ), Av. España 1512 (Sur), J5402DSP San Juan, Argentina
}

Received 1 September 2018 / Accepted 27 September 2018

\begin{abstract}
Context. In spite of the numerous studies of low-luminosity galaxies in different environments, there is still no consensus about their formation scenario. In particular, a large number of galaxies displaying extremely low surface brightnesses have been detected in recent years and the nature of these objects is still under discussion.

Aims. In order to enlarge the sample of known low-surface-brightness (LSB) galaxies and to try to provide clues about their nature, we report the detection of eight such objects $\left(\mu_{\mathrm{eff}, g^{\prime}} \simeq 27 \mathrm{mag} \mathrm{arcsec}^{-2}\right)$ towards the group of galaxies Pegasus I. They are located, in projection, within a radius of $\sim 200 \mathrm{kpc}$ in the very center of Pegasus I, close to the dominant elliptical galaxies NGC 7619 and NGC 7626.

Methods. We analyzed deep, high-quality GEMINI-GMOS images with ELLIPSE within IRAF in order to obtain their brightness profiles and structural parameters. We also fit Sérsic functions to these profiles in order to compare their properties with those of typical early-type galaxies.

Results. Assuming that these galaxies are at the distance of Pegasus I, we have found that their sizes are intermediate among similar objects reported in the literature. In particular, we found that three of these galaxies can be classified as ultra-diffuse galaxies and a fourth one displays a nucleus. The eight new LSB galaxies show significant color dispersion around the extrapolation towards faint luminosities of the color-magnitude relation defined by typical early-type galaxies. In addition, they display values of the Sérsic index below 1 (concave brightness profiles in linear scale), in agreement with values obtained for LSB galaxies in other environments. Conclusions. We show that there seems to be a bias effect in the size distributions of the detected LSBs in different environments, in the sense that more distant groups/clusters lack small $r_{\text {eff }}$ objects, while large systems are not found in the Local Group and nearby environments. While there may be an actual shortage of large LSB galaxies in low-density environments like the Local Group, the non-detection of small (and faint) systems at large distances is clearly a selection effect. As an example, LSB galaxies with similar sizes to those of the satellites of Andromeda in the Local Group will be certainly missed in a visual identification at the distance of Pegasus I.
\end{abstract}

Key words. methods: observational - techniques: photometric - galaxies: groups: individual: Pegasus I galaxies: star clusters: general - galaxies: dwarf

\section{Introduction}

The "cloud of nebulae" extending through the Pegasus constellation (Pegasus I; Zwicky 1942; Zwicky et al. 1965) was initially reported to be a "medium compact cluster with an angular diameter of 6.3 degrees", containing 30 confirmed members, with a similar number of spiral and early-type galaxies spanning a radial velocity range of $2500-5500 \mathrm{~km} \mathrm{~s}^{-1}$ (Chincarini \& Rood 1976; Aaronson et al. 1980, and references therein). From an H I study of 80 galaxies in the Pegasus I region, (Richter \& Huchtmeier 1982) increased the number of confirmed members to 75 bright galaxies located at $51.8 \mathrm{Mpc}$, with a mean radial velocity of $3885 \mathrm{~km} \mathrm{~s}^{-1}$ and a small velocity dispersion of $236 \mathrm{~km} \mathrm{~s}^{-1}$. This distance is in agreement with the value of $53 \mathrm{Mpc}$ obtained more recently by Tonryetal. (2001) through the application of the surface-brightness fluctuations method.
The Pegasus I group is dominated by two giant elliptical galaxies of similar luminosity: $\operatorname{NGC7619}(V=11.06 \mathrm{mag})$ and NGC 7626 ( $V=11.08$ mag; de Vaucouleurs et al. 1992). Both galaxies are radio sources and show morphological peculiarities. NGC 7626 presents symmetric radio-jets/lobes on each side of its central core, outer and inner shells, a nuclear dust lane, and a compact X-ray source at its center, among other features (Birkinshaw \& Davies 1985; Jedrzejewski \& Schechter 1988; Balcells \& Carter 1993; Trinchieri et al. 1997). NGC 7619 on the other hand is a strong and extended X-ray source with a long asymmetric X-ray tail in the southwest direction (Kim et al. 2008; Randall et al. 2009) that has been interpreted as being due to the infall of this galaxy into Pegasus I. In addition, cold fronts detected in each of these dominant galaxies using Chandra images (Randall et al. 2009) support the scenario of a major merger of two subgroups. It is worth noticing that Pegasus I 
was earlier reported as presenting an excess of star-forming early-type galaxies in its outskirts, akin to Butcher-Oemler clusters (Vigroux et al. 1989; Trentham 1997). This excess would be associated with the infall of galaxies into Pegasus I, which would indicate that it is a group in formation (see also, Fujita 2003).

Canizares et al. (1986) report an intergalactic gas density of $2 \times 10^{-4} \mathrm{~cm}^{-3}$ for Pegasus I, making it one of the least dense media ever detected in a group of galaxies. From this fact and the low-velocity dispersion of the group, ram-pressure stripping would not be expected to play a key role in driving its galaxy evolution. However, Levy et al. (2007) found that, though not as intense as in rich clusters, there is gas depletion in a significant number of late-type galaxies. This would indicate that the interaction between the interstellar medium and the intracluster medium may indeed be playing an important role in Pegasus I.

The faint galaxy content of Pegasus I has been poorly studied. Vader \& Chaboyer (1994) presented surface photometry of seven galaxies displaying dwarf morphologies located in the neighborhoods of NGC 7626, but with no radial velocity information at that moment. A few years later, Trentham (1997) attempted a determination of the faint end of the galaxy luminosity function in Pegasus I; however, the low galaxy density in his sampled fields resulted in a relatively small number of objects brighter than $M_{R}>-11$, thus leaving the group luminosity function essentially unconstrained. More recently, through a friends-of-friends (FoF) algorithm, Ramella et al. (2002) identified Pegasus I as a physical association with 13 spectroscopically confirmed members.

In this context, we have started the study of the faint galaxy content of the central region of Pegasus I through deep optical images obtained with the telescope of the Gemini North Observatory. In this paper (the first of a series) we present a photometric study of eight low-surfacebrightness (LSB) galaxies detected towards the central region of Pegasus I. Originally identified in the Local Group (LG), LSB galaxies are extended objects displaying central surface brightnesses $\mu_{0, B}>23 \mathrm{mag} \operatorname{arcsec}^{-2}$ (Impey \& Bothun 1997). This characteristic makes their detection and subsequent analysis a challenging task. The interest in the detection of such extremely faint galaxies outside the LG has increased in recent years, as they are expected to impose strong constraints on models of galaxy formation and evolution. Some questions arise when analyzing these objects: are these extragalactic systems the counterparts of the LG dwarf spheroidal (dSph) galaxies which are supposed to be the most dark matter (DM)dominated systems in the near universe? Are they formed in-situ, or are they kinematically decoupled and gravitationally bound structures arising from the interaction of massive galaxies that, as a consequence, are not expected to contain DM at all (Dabringhausen \& Kroupa 2013, and references there in)? Previous explorations of these subjects regarding Pegasus I have been made with small telescopes by O'Neil et al. (1997) and Shi et al. (2017). None of the galaxies presented in this paper were included in those works. Therefore, our sample contributes to increasing the number of LSB galaxies identified towards Pegasus I.

The paper is organized as follows. In Sect. 2 we describe the photometric data. In Sects. 3 and 4, we present the strategies followed to obtain photometric parameters. In Sect. 5 we present the results obtained from our analysis and in Sect. 6 we provide a discussion and our conclusions.

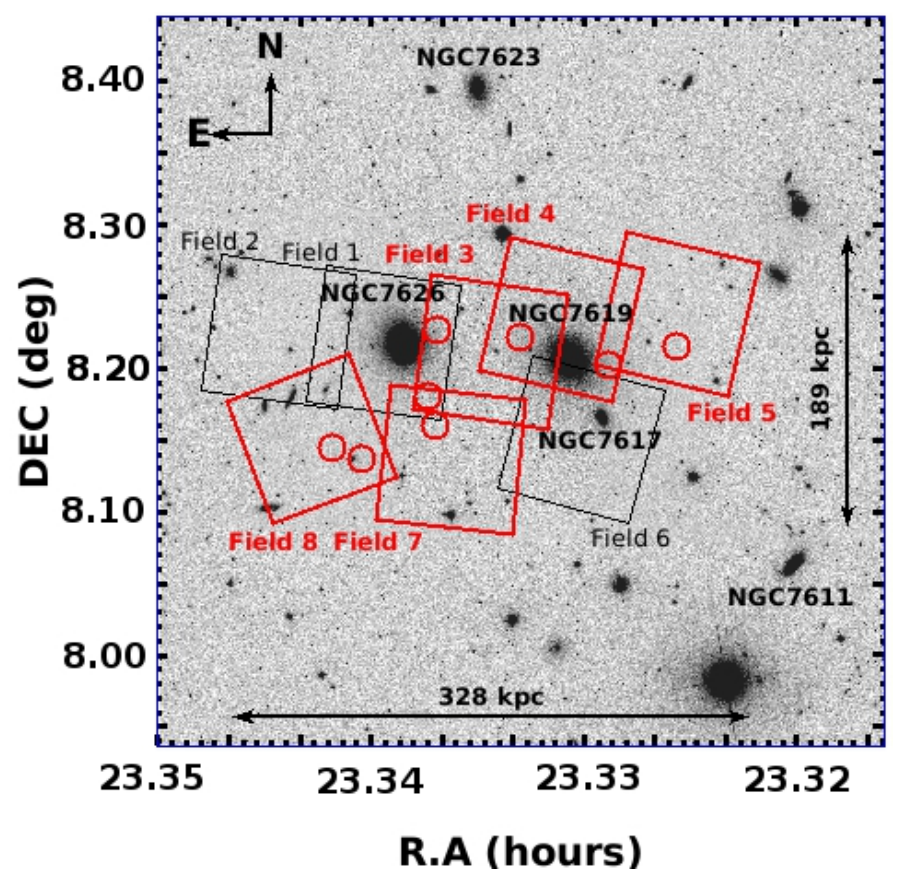

Fig. 1. $30.5 \times 30.5$ arcmin mosaic in the $r^{\prime}$ filter obtained from the SDSS database (DR12) showing the central region of Pegasus I. Red squares depict the Gemini-GMOS frames used in this work $(\sim 5.5 \operatorname{arcmin}$ on a side). Their designation corresponds to the order in which each frame was observed during our Gemini survey of Pegasus I. Red circles indicate the location of the LSB objects. The large arrows depict the spatial scales covered by our Gemini-GMOS fields. North is up and east is to the left.

\section{Observational data}

We have obtained deep $g^{\prime}, r^{\prime}$, and $i^{\prime}$ images of eight fields in the central region of Pegasus I, with the Gemini Multi-Object Spectrograph (GMOS; Hook et al. 2004) of GEMINI-North, during semesters 2008B, 2012A, 2014A, 2014B and 2015B. These observations are part of an ongoing survey of the globular clusters population in the central region of Pegasus I with the Gemini telescopes (Faifer et al., in prep.). In five of those fields we identified eight LSB galaxies. Figure 1 shows the location of the eight observed fields, and the projected spatial distribution of the detected objects in the central region of Pegasus I.

GMOS consists of three CCDs with $2048 \times 4096$ pixels each, separated by $\sim 2.8$ arcsec gaps, and with an unbinned pixel scale of $0.0727 \mathrm{arcsec}_{\mathrm{pixel}}{ }^{-1}$. The field of view (FOV) is $5.5 \times 5.5$ arcmin and the scale for binning $2 \times 2$ is 0.146 arcsec pixel $^{-1}$. In Table 1 we show the information related to the GEMINI-GMOS images.

We performed spatial dithering between the individual exposures in order to facilitate the removal of cosmic rays and to fill in the gaps between the CCD chips. The raw images were processed using the GMOS package within IRAF (e.g., gprepare, gbias, giflat, gireduce and gmosaic). The appropriate bias and flat-field images were obtained from the Gemini Science Archive (GSA) as part of the standard GMOS baseline calibrations.

As previously mentioned, the data used in this work were obtained over several semesters. For that reason, some fields were observed with the GMOS-N E2V DD CCDs (previous to their replacement by Hamamatsu CCDs), thus displaying strong fringing patterns in the $i^{\prime}$ frames. These patterns were removed by subtracting a master fringe frame that was created using gifringe and girmfringe task. 
Table 1. Log of the GEMINI-GMOS images.

\begin{tabular}{|c|c|c|c|c|c|c|c|}
\hline Field & Program & $\begin{array}{c}\mathrm{RA} \\
(\mathrm{J} 2000)\end{array}$ & $\begin{array}{c}\text { Dec } \\
(\mathrm{J} 2000)\end{array}$ & Filter & Date & $\begin{array}{c}\text { Exposures } \\
\text { (s) }\end{array}$ & $\begin{array}{l}F W H M \\
(\operatorname{arcsec})\end{array}$ \\
\hline 1 & GN-2008B-Q-14 & $23: 20: 46.13$ & 08:13:05.9 & $\begin{array}{l}g^{\prime} \\
r^{\prime} \\
i^{\prime}\end{array}$ & $\begin{array}{l}4 \text { Aug. } 2008 \\
4 \text { Aug. } 2008 \\
4 \text { Aug. } 2008\end{array}$ & $\begin{array}{l}8 \times 450 \\
4 \times 450 \\
4 \times 450\end{array}$ & $\begin{array}{l}0.9 \\
0.8 \\
0.8\end{array}$ \\
\hline 2 & GN-2008B-Q-14 & $23: 21: 03.85$ & $08: 13: 31.6$ & $\begin{array}{l}g^{\prime} \\
r^{\prime} \\
i^{\prime}\end{array}$ & $\begin{array}{l}8 \text { Aug. } 2008 \\
8 \text { Aug. } 2008 \\
8 \text { Aug. } 2008\end{array}$ & $\begin{array}{l}8 \times 450 \\
4 \times 450 \\
4 \times 450\end{array}$ & $\begin{array}{l}0.8 \\
0.7 \\
0.7\end{array}$ \\
\hline 3 & GN-2008B-Q-14 & $23: 20: 27.82$ & $08: 12: 41.3$ & $\begin{array}{l}g^{\prime} \\
r^{\prime} \\
i^{\prime}\end{array}$ & $\begin{array}{l}\text { 10 Aug. } 2008 \\
10 \text { Aug. } 2008 \\
\text { 10 Aug. } 2008\end{array}$ & $\begin{array}{l}8 \times 450 \\
4 \times 450 \\
4 \times 450\end{array}$ & $\begin{array}{l}0.8 \\
0.8 \\
0.9\end{array}$ \\
\hline 4 & GN-2008B-Q-14 & $23: 20: 15.62$ & 08:14:00.8 & $\begin{array}{l}g^{\prime} \\
r^{\prime} \\
i^{\prime}\end{array}$ & $\begin{array}{l}8 \text { Aug. } 2008 \\
8 \text { Aug. } 2008 \\
8 \text { Aug. } 2008\end{array}$ & $\begin{array}{l}8 \times 450 \\
4 \times 450 \\
4 \times 450\end{array}$ & $\begin{array}{l}0.9 \\
1.0 \\
0.7\end{array}$ \\
\hline 5 & GN-2012A-Q-55 & 23:19:56.08 & 08:14:14.9 & $\begin{array}{l}g^{\prime} \\
r^{\prime} \\
i^{\prime}\end{array}$ & $\begin{array}{l}\text { 23 Jun. } 2012 \\
\text { 21 Jun. } 2012 \\
\text { 24 Jun. } 2012\end{array}$ & $\begin{array}{l}8 \times 440 \\
4 \times 440 \\
6 \times 300\end{array}$ & $\begin{array}{l}0.8 \\
0.7 \\
0.8\end{array}$ \\
\hline 6 & GN-2014A-Q-70 & $23: 20: 12.68$ & 08:08:58.91 & $\begin{array}{l}g^{\prime} \\
r^{\prime} \\
i^{\prime}\end{array}$ & $\begin{array}{l}23 \text { Jun. } 2014 \\
25 \text { Jun. } 2014 \\
25 \text { Jun. } 2014\end{array}$ & $\begin{array}{l}8 \times 450 \\
4 \times 450 \\
6 \times 300\end{array}$ & $\begin{array}{l}0.7 \\
0.6 \\
0.5\end{array}$ \\
\hline 7 & GN-2014B-Q-17 & $23: 20: 34.10$ & 08:08:06.1 & $\begin{array}{l}g^{\prime} \\
r^{\prime} \\
i^{\prime}\end{array}$ & $\begin{array}{l}27 \text { Jul. } 2014 \\
27 \text { Jul. } 2014 \\
27 \text { Jul. } 2014\end{array}$ & $\begin{array}{l}8 \times 450 \\
4 \times 450 \\
6 \times 300\end{array}$ & $\begin{array}{l}1.0 \\
0.9 \\
0.9\end{array}$ \\
\hline 8 & GN-2015B-Q-13 & $23: 20: 58.08$ & 08:09:03.9 & $\begin{array}{l}g^{\prime} \\
r^{\prime} \\
i^{\prime}\end{array}$ & $\begin{array}{l}25 \text { Jul. } 2015 \\
25 \text { Jul. } 2015 \\
25 \text { Jul. } 2015\end{array}$ & $\begin{array}{l}10 \times 450 \\
8 \times 300 \\
8 \times 300\end{array}$ & $\begin{array}{l}0.6 \\
0.6 \\
0.5\end{array}$ \\
\hline
\end{tabular}

Table 2. Distance information of the dominant galaxies of Pegasus I.

\begin{tabular}{cccccccc}
\hline Galaxy & $\begin{array}{c}\text { RA } \\
(\mathrm{J} 2000)\end{array}$ & $\begin{array}{c}\text { Dec } \\
(\mathrm{J} 2000)\end{array}$ & $\begin{array}{c}(m-M) \\
(\mathrm{mag})\end{array}$ & $\begin{array}{c}\text { Distance } \\
(\mathrm{Mpc})\end{array}$ & Method & $\begin{array}{c}\mathrm{H}_{0} \\
\left(\mathrm{~km} \mathrm{~s}^{-1} \mathrm{Mpc}^{-1}\right)\end{array}$ & Reference \\
\hline NGC 7619 & $23: 20: 14.5$ & $08: 12: 22$ & 33.70 & 55.0 & SBF & 74.4 & Tully et al. (2013) \\
NGC 7626 & $23: 20: 42.5$ & $08: 13: 01$ & 33.34 & 46.6 & SBF & 74.4 & Tully et al. (2013) \\
\hline
\end{tabular}

Notes. SBF: surface brightness fluctuations.

The resulting images corresponding to the same filter and the same field obtained from the above procedure were finally co-added using the task imcoadd in order to obtain the images used for further analysis. The photometric data obtained from them were later calibrated to the photometric system of the Sloan Digital Sky Survey (SDSS).

\section{Sky subtraction and detection of LSB galaxies}

From visual inspection of our GEMINI-GMOS images we identified eight diffuse and extended objects (Table 3). In order to obtain their brightness profiles and their photometric parameters, we had to subtract the contribution of the sky to the images. It is important to note that the average surface brightness of the sky in our images is $\mu_{\mathrm{sky}, g^{\prime}} \simeq 22 \mathrm{mag} \mathrm{arcsec}^{-2}$, $\mu_{\text {sky }, r^{\prime}} \simeq 21 \mathrm{magarcsec}^{-2}, \mu_{\text {sky }, i^{\prime}} \simeq 20 \mathrm{magarcsec}^{-2}$, while the objects of our study display central surface brightnesses much fainter than these values $\left(\mu_{g^{\prime}} \gtrsim 25 \mathrm{mag} \operatorname{arcsec}^{-2}\right)$. Therefore, to obtain reliable photometric and structural parameters, it is necessary to perform a careful modeling and subtraction of the background. The general process consisted of modeling the surface brightness distributions of the elliptical galaxies NGC 7626 and
NGC 7619 and their respective halos, including several extended objects that could affect the surface brightness profiles. One difficulty with this process was that the halos of the two elliptical galaxies extended beyond the edge of each individual field. The different strategies followed in each field to achieve this goal are outlined below.

Field 3: The subtraction of the halos of the bright elliptical galaxies NGC7619 and NGC7626, combined with SExtractor. After subtracting preliminary constant values of the sky level from the original images, we modeled the halos of the galaxies through the task ELLIPSE within IRAF (Tody 1986; Jedrzejewski 1987). These models were subtracted from the skysubtracted images, but the residuals still showed significant systematic variations in the regions of the LSB galaxies. As the photometric parameters of this type of galaxy are very sensitive to sky variations, we used SExtractor (Bertin \& Arnouts 1996) to build a model of this residual background, which was then subtracted from the halos-subtracted frames.

Field 4: The subtraction of models of NGC 7619, extended objects, and bright stars present in the field. After subtracting a preliminary mean sky value from the images, we modeled the light of NGC 7619 through ELLIPSE. Other extended objects 
and bright stars, in the region of the detected LSB galaxies were also modeled and subtracted in order to diminish any remaining background variation. After a couple of iterations in the modeling/subtraction process, we obtained satisfactorily flat frames (the mean residual value is lower than $0.5 \%$ of the original value) in the regions of the LSB galaxies.

Field 5: The subtraction of the halo of NGC 7619 combined with SExtractor. The procedure in this case was the same as for Field 3.

Field 7: The subtraction of the halo of NGC 7626. We used here a similar procedure to that applied to Field 3 but in this case SExtractor was not needed to improve the background behavior in the regions of the LSB galaxies (the mean residual value is lower than $0.25 \%$ of the original value).

Field 8: The subtraction of the models of extended objects located near the LSB galaxies. We worked similarly as for Field 4 , except that these images were not affected by the halos of the bright ellipticals.

\section{Surface brightness profiles}

\subsection{Model-independent parameters}

In order to obtain the brightness profiles of the LSB galaxies detected in our frames, we worked with ELLIPSE within IRAF on the GEMINI-GMOS sky-subtracted and registered $g^{\prime}, r^{\prime}$ and $i^{\prime}$ images. In all the images, these objects display smooth and diffuse morphologies without showing inner substructure (bars, dust bands, etc.) or color gradients in their color maps. Therefore, we were able to add the $g^{\prime}, r^{\prime}$ and $i^{\prime}$ images corresponding to the same field in order to improve the signal-to-noise ratio $(\mathrm{S} / \mathrm{N})$ by enhancing the extremely low surface brightnesses of these galaxies, therefore allowing ELLIPSE to converge with as many free parameters as possible. The isophote tables obtained from the added images were used as a reference to get the tables corresponding to each individual filter.

The instrumental brightness profiles were corrected by the residual sky level through the analysis of the curves of growth (COGs; for details, we refer the reader to Sect. 3.1 of Smith Castelli et al. 2016) and, later, calibrated to the SDSS photometric system. To do so, we used standard star fields observed within our programs. The expression used to calibrate our data was:

$\mu_{\text {std }}=\mu_{\text {zero }}+\mu_{\text {inst }}+2.5 \log \left(s c a^{2} t_{\text {exp }}\right)-K(X-1.0)$,

where $\mu_{\text {std }}$ is the surface brightness (in standard magnitudes $\left.\operatorname{arcsec}^{-2}\right), \mu_{\text {zero }}$ is the photometric zero-point, $\mu_{\text {inst }}$ is the instrumental surface brightness, $s c a$ is the scale of the images, $t_{\exp }$ is the exposure time, $K$ is the mean atmospheric extinction at Mauna Kea, and $X$ is the airmass value.

The upper panels of Fig. 2 show the $r^{\prime}$ image of one of the LSB galaxies detected in Pegasus I (left), and the residuals left by the model subtraction (right; see Sect. 4.2). Similar figures for the rest of Pegasus' LSB galaxies can be found in the appendix.

In Table 3 we present the model-independent photometric parameters obtained from the $g^{\prime}, r^{\prime}$ and $i^{\prime}$ observed brightness profiles. In this case, total magnitudes are obtained through the numerical integration of these profiles up to the total equivalent radius $\left(r_{\text {tot }}\right)$ of the galaxy (see Table 3 ). We consider that $r_{\text {tot }}$ is the equivalent radius at which the curve of growth stabilizes in the three filters. The corresponding errors were calculated from the integration of the brightness profiles obtained after adding and subtracting the median value of the residual sky level to the original profile.

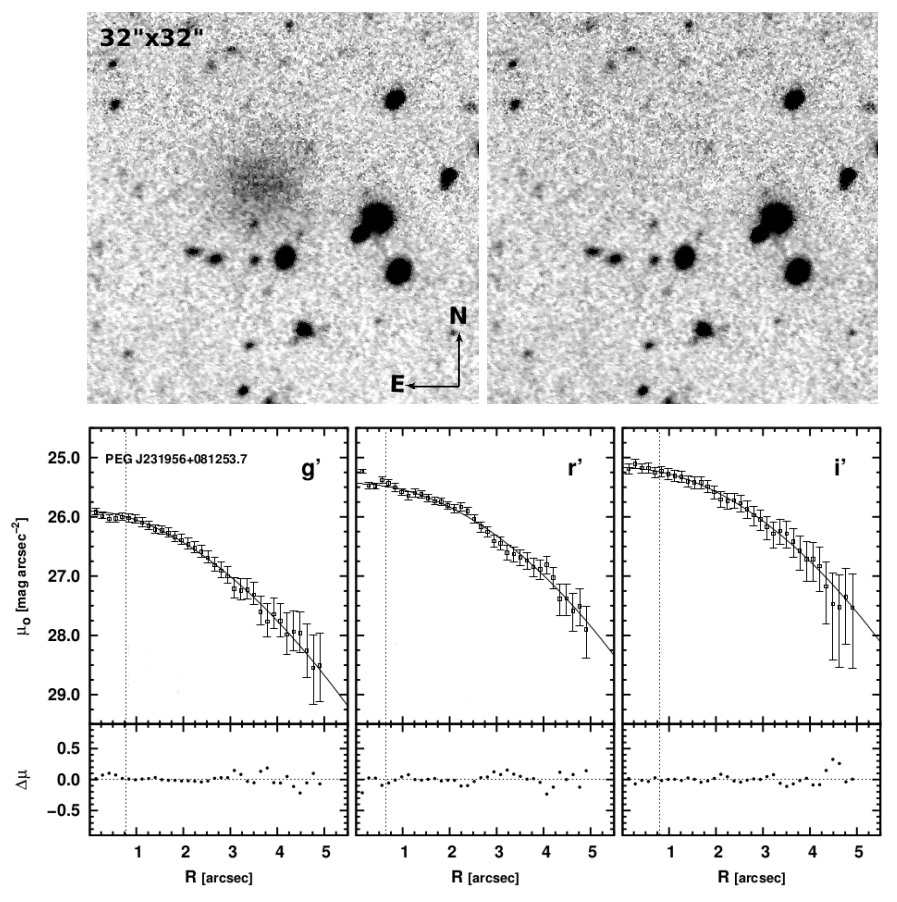

Fig. 2. Upper panels: $32 \times 32$ arcsec frame in the $r^{\prime}$ filter showing the LSB galaxy PEGJ231956+081253.7 (left) and the residuals left by the subtraction of the model built from the brightness profile obtained with ELLIPSE (right). Lower panels: Sérsic law fits to the absorption corrected $g^{\prime}, r^{\prime}$ and $i^{\prime}$ brightness profiles of the LSB galaxy PEG J231956+081253.7. The residuals $\Delta \mu=\mu$ (obs) $-\mu$ (fit) are shown in the lower panel. The dotted vertical lines indicate the inner region of the profiles excluded to perform the fits in order to avoid seeing effects.

\subsection{Sérsic profiles}

Due to the extremely low surface brightnesses of our objects, we would like to compare our model-independent parameters with those obtained from fitting a general Sérsic law (Sérsic 1963) to their surface brightness profiles:

$\mu(r)=\mu_{0}+1.0857\left(\frac{r}{r_{0}}\right)^{N}$.

Here, $\mu_{0}$ designates the central $(r=0)$ surface brightness, $r_{0}$ is the scale length of the profile and $N$ is the Sérsic index. Due to its simpler mathematical dependence on the free parameters, we decided to use the above formula instead of

$\mu(r)=\mu_{\mathrm{eff}}+1.0857 b_{n}\left[\left(\frac{r}{r^{\mathrm{s}}{ }_{\mathrm{eff}}}\right)^{\frac{1}{n}}-1\right]$,

where $b_{n} \simeq 1.9992 n-0.3271$ for $0.5<n<10$ (Graham \& Worley 2008, and references therein) and the superscript $s$ refers to quantities obtained assuming a Sérsic profile. There are simple relations between the quantities involved in both equations (e.g., MacArthur et al. 2003):

$n=\frac{1}{N}$

$\mu_{\mathrm{eff}}=\mu_{0}+1.0857 b_{n}$

$r_{\mathrm{eff}}^{\mathrm{s}}=r_{0} b_{n}^{n}$.

All galaxies except PEG J232054+080838.8 (Fig. A.7) were fitted with a single Sérsic law, excluding the inner regions 
N. M. González et al.: Stellar systems in the direction of Pegasus I. I. LSB galaxies

Table 3. Model-independent photometric parameters of the low-surface brightness objects presented in this paper.

\begin{tabular}{|c|c|c|c|c|c|c|c|c|}
\hline $\begin{array}{l}\text { Object } \\
\text { (1) }\end{array}$ & $\begin{array}{c}\text { RA } \\
(\mathrm{J} 2000) \\
(2)\end{array}$ & $\begin{array}{c}\text { Dec } \\
(\mathrm{J} 2000) \\
(3)\end{array}$ & $\begin{array}{l}\text { Filter } \\
(4)\end{array}$ & $\begin{array}{c}r_{\text {tot }} \\
(\operatorname{arcsec}) \\
(5)\end{array}$ & $\begin{array}{c}r_{\mathrm{eff}} \\
(\operatorname{arcsec}) \\
(6)\end{array}$ & $\begin{array}{c}m_{\mathrm{tot}} \\
(\mathrm{mag}) \\
(7)\end{array}$ & $\begin{array}{c}\left(g^{\prime}-r^{\prime}\right) \\
(\mathrm{mag}) \\
(8)\end{array}$ & $\begin{array}{c}\left\langle\mu_{\mathrm{eff}}\right\rangle \\
\left(\mathrm{mag} \mathrm{arcsec}^{-2}\right) \\
(9)\end{array}$ \\
\hline PEG J231956+081253.7 & $23: 19: 56$ & $08: 12: 53.7$ & $\begin{array}{l}g^{\prime} \\
r^{\prime} \\
i^{\prime}\end{array}$ & 7.15 & $\begin{array}{l}2.52_{-0.28}^{+0.28} \\
2.66_{-0.14}^{+0.14} \\
2.66_{-0.42}^{+0.42} \\
\end{array}$ & $\begin{array}{l}22.53_{-0.28}^{+0.20} \\
21.69_{-0.15}^{+0.16} \\
21.48_{-0.29}^{+0.33} \\
\end{array}$ & $0.83_{-0.31}^{+0.26}$ & $\begin{array}{l}26.53_{-0.39}^{+0.30} \\
25.82_{-0.19}^{+0.19} \\
25.60_{-0.50}^{+0.44} \\
\end{array}$ \\
\hline PEG J232023+081331.4 & $23: 20: 23$ & $08: 13: 31.4$ & $\begin{array}{l}g^{\prime} \\
r^{\prime} \\
i^{\prime}\end{array}$ & 19.15 & $\begin{array}{l}7.78_{-2.39}^{+4.79} \\
8.38_{-1.80}^{+2.39} \\
7.78_{-1.19}^{+2.99}\end{array}$ & $\begin{array}{l}21.09_{-0.65}^{+0.92} \\
19.97_{-0.47}^{+0.71} \\
19.70_{-0.50}^{+0.63}\end{array}$ & $1.11_{-0.80}^{+1.17}$ & $\begin{array}{l}27.54_{-1.22}^{+1.27} \\
26.58_{-0.77}^{+0.86} \\
26.16_{-0.65}^{+0.88}\end{array}$ \\
\hline PEG J232024+081209.0 & $23: 20: 24$ & 08:12:09.0 & $\begin{array}{l}g^{\prime} \\
r^{\prime} \\
i^{\prime}\end{array}$ & 16.35 & $\begin{array}{l}8.30_{-0.11}^{+1.18} \\
7.58_{-1.18}^{+2.13} \\
7.82_{-0.95}^{+1.89}\end{array}$ & $\begin{array}{l}20.27_{-0.34}^{+0.45} \\
19.53_{-0.44}^{+0.62} \\
19.66_{-0.44}^{+0.61}\end{array}$ & $0.74_{-0.55}^{+0.77}$ & $\begin{array}{r}26.86_{-0.39}^{+0.52} \\
25.93_{-0.60}^{+0.79} \\
26.12_{-0.53}^{+0.75}\end{array}$ \\
\hline PEG J232037+080934.3 & $23: 20: 37$ & 08:09:34.3 & $\begin{array}{l}g^{\prime} \\
r^{\prime} \\
i^{\prime}\end{array}$ & 16.94 & $\begin{array}{l}5.67_{-1.16}^{+1.54} \\
5.02_{-1.28}^{+1.20} \\
5.02_{-1.28}^{+0.89}\end{array}$ & $\begin{array}{l}20.34_{-0.35}^{+0.44} \\
19.93_{-0.35}^{+0.40} \\
19.60_{-0.31}^{+0.35}\end{array}$ & $0.40_{-0.50}^{+0.60}$ & $\begin{array}{l}26.10_{-0.67}^{+0.64} \\
25.43_{-0.85}^{+0.59} \\
25.10_{-0.83}^{+0.48}\end{array}$ \\
\hline PEG J232037+081336.6 & $23: 20: 37$ & $08: 13: 36.6$ & $\begin{array}{l}g^{\prime} \\
r^{\prime} \\
i^{\prime}\end{array}$ & 7.17 & $\begin{array}{l}2.17_{-0.33}^{+0.24} \\
2.35_{-0.15}^{+0.18} \\
2.50_{-0.52}^{+0.43}\end{array}$ & $\begin{array}{l}22.36_{-0.27}^{+0.21} \\
21.62_{-0.15}^{+0.12} \\
21.16_{-0.33}^{+0.37} \\
\end{array}$ & $0.74_{-0.30}^{+0.24}$ & $\begin{array}{l}26.04_{-0.48}^{+0.30} \\
25.47_{-0.21}^{+0.20} \\
25.16_{-0.68}^{+0.49} \\
\end{array}$ \\
\hline PEG J232038+081046.9 & $23: 20: 38$ & 08:10:46.9 & $\begin{array}{l}g^{\prime} \\
r^{\prime} \\
i^{\prime}\end{array}$ & 9.26 & $\begin{array}{l}2.72_{-0.82}^{+0.77} \\
2.72_{-1.06}^{+1.06} \\
2.99_{-1.09}^{+1.33}\end{array}$ & $\begin{array}{l}21.53_{-0.33}^{+0.38} \\
20.99_{-0.40}^{+0.33} \\
20.62_{-0.45}^{+0.40}\end{array}$ & $0.53_{-0.52}^{+0.47}$ & $\begin{array}{l}25.70_{-1.05}^{+0.59} \\
25.16_{-1.79}^{+0.71} \\
25.00_{-1.48}^{+0.80}\end{array}$ \\
\hline PEG J232049+080806.2 & $23: 20: 49$ & 08:08:06.2 & $\begin{array}{l}g^{\prime} \\
r^{\prime} \\
i^{\prime}\end{array}$ & 9.71 & $\begin{array}{l}3.07_{-0.92}^{+0.92} \\
2.96_{-1.43}^{+1.43} \\
3.78_{-1.02}^{+1.43}\end{array}$ & $\begin{array}{l}22.02_{-0.33}^{+0.40} \\
21.53_{-0.50}^{+0.61} \\
20.80_{-0.36}^{+0.48}\end{array}$ & $0.49_{-0.60}^{+0.73}$ & $\begin{array}{l}26.45_{-1.05}^{+0.65} \\
25.88_{-3.96}^{+0.96} \\
25.67_{-0.91}^{+0.78}\end{array}$ \\
\hline PEG J232054+080838.8 & $23: 20: 54$ & 08:08:38.8 & $\begin{array}{l}g^{\prime} \\
r^{\prime} \\
i^{\prime}\end{array}$ & 10.93 & $\begin{array}{l}3.99_{-1.06}^{+1.99} \\
3.99_{-0.80}^{+1.33} \\
4.13_{-1.06}^{+2.13}\end{array}$ & $\begin{array}{l}21.69_{-0.53}^{+0.58} \\
21.10_{-0.40}^{+0.46} \\
20.70_{-0.57}^{+0.67}\end{array}$ & $0.59_{-0.66}^{+0.74}$ & $\begin{array}{l}26.70_{-0.98}^{+0.95} \\
26.11_{-0.68}^{+0.72} \\
25.78_{-0.97}^{+1.02}\end{array}$ \\
\hline
\end{tabular}

Notes. They were obtained from the analysis of the COGs. Apparent magnitudes and colors have not been corrected for extinction or reddening. Column (5): $r_{\text {tot }}$ is the equivalent radius $(r=\sqrt{a b}=a \sqrt{1-\epsilon})$ at which the curve of growth stabilizes in the three filters. Column (6): $r_{\text {eff }}$ is the equivalent radius that contains half of the flux within $r_{\text {tot }}$. Column (7): total integrated magnitudes are calculated from the numerical integration of the observed brightness profiles up to $r_{\text {tot }}$. Column (8): $\left\langle\mu_{\text {eff }}\right\rangle=\operatorname{mag}+2.5 \log \left(2 \pi r_{\text {eff }}^{2}\right)$.

affected by seeing. In each case, the fit extended from an inner radius defined by the seeing FWHM, to a maximum radius set at the point where the profile begins to be significantly affected by small variations in the sky level.

PEG J232054+080838.8 displays what seems to be a nucleus. As a first step, we built a PSF model of this starlike object with DAOPHOT within IRAF. After subtracting this model to the original images of the galaxy, the $g^{\prime}, r^{\prime}$, and $i^{\prime}$ frames displayed some residuals. This was taken as an evidence that the nucleus is marginally resolved in all the images. Therefore, we decided to fit the sum of two Sérsic functions to the whole $g^{\prime}, r^{\prime}$ and $i^{\prime}$ profiles, without excluding the very inner region, with the aim of obtaining structural parameters for the central component. We tested the results of the composite fit, first considering an external component with $n=1$ and then a fit with free parameters for both components. We have found that allowing all the parameters to vary freely gives the minimum $\chi^{2}$.

In Table 4 we present the resulting structural parameters, as well as the internal and external radii considered to perform the fits in each case (Cols. 4 and 5, respectively). Total integrated magnitudes were also obtained from the expression

$$
\begin{aligned}
m_{\text {tot }}^{\mathrm{s}} & =\mu_{\mathrm{eff}}-1.995450-5 \log \left(r^{\mathrm{s}}{ }_{\text {eff }}\right) \\
& -1.0857 b_{n}-2.5 \log \left[b_{n}^{-2 n} n \Gamma(2 n)\right] .
\end{aligned}
$$

From Tables 3 and 4, it can be seen that Sérsic magnitudes and $r_{\text {eff }}$ are in agreement with model-independent ones, within the errors. As an example, in the lower panels of Fig. 2, we show the fits to the brightness profiles and their residuals for one of the LSB galaxies detected in Pegasus I. Similar figures for the rest of Pegasus' LSB galaxies are shown in the appendix.

\section{Comparison with other samples}

\subsection{Color-magnitude diagram}

It is interesting to compare the photometric properties of the LSB galaxies detected in the direction of Pegasus I with those of similar objects identified in other environments. To this aim, in Fig. 3 we present two color-magnitude diagrams (CMDs) showing the location of dwarf spheroidal (dSph), LSB, and ultradiffuse galaxies (UDGs) reported in the literature, along with the LSB galaxies analyzed in this work, assuming that the latter are at the distance of Pegasus I. We consider the distance modulus of Pegasus I as that obtained from the mean distance of 
Table 4. Structural parameters of the LSB galaxy candidates in Pegasus I, obtained from the fit of Sérsic laws to their brightness profiles.

\begin{tabular}{|c|c|c|c|c|c|c|c|c|c|c|}
\hline $\begin{array}{c}\text { Object } \\
\text { (1) }\end{array}$ & $\begin{array}{l}\text { Filter } \\
\text { (2) }\end{array}$ & $\begin{array}{c}r_{\text {int }} \\
(\operatorname{arcsec}) \\
(3)\end{array}$ & $\begin{array}{c}r_{\mathrm{ext}} \\
(\operatorname{arcsec}) \\
(4)\end{array}$ & $\begin{array}{c}\mu_{0} \\
\left(\mathrm{mag} \operatorname{arcsec}^{-2}\right) \\
(5)\end{array}$ & $\begin{array}{c}r_{0} \\
(\operatorname{arcsec}) \\
(6)\end{array}$ & $\begin{array}{l}\mathrm{N} \\
\text { (7) }\end{array}$ & $\begin{array}{c}\mu_{\mathrm{eff}} \\
\left(\mathrm{mag} \operatorname{arcsec}^{-2}\right) \\
(8)\end{array}$ & $\begin{array}{c}r_{\text {eff }}^{s} \\
(\operatorname{arcsec}) \\
(9)\end{array}$ & $\begin{array}{c}n \\
(10)\end{array}$ & $\begin{array}{c}m^{\mathrm{s}} \text { tot } \\
(\mathrm{mag}) \\
(11)\end{array}$ \\
\hline \multirow{3}{*}{ PEG J231956+081253.7 } & $g^{\prime}$ & 0.8 & \multirow{3}{*}{5} & $25.90 \pm 0.03$ & $2.97 \pm 0.05$ & $1.80 \pm 0.07$ & 26.75 & 2.60 & 0.56 & 22.23 \\
\hline & $r^{\prime}$ & 0.7 & & $25.44 \pm 0.03$ & $3.34 \pm 0.07$ & $1.97 \pm 0.13$ & 26.19 & 2.77 & 0.51 & 21.57 \\
\hline & $i^{\prime}$ & 0.8 & & $25.17 \pm 0.02$ & $3.30 \pm 0.05$ & $1.95 \pm 0.09$ & 25.93 & 2.74 & 0.51 & 21.33 \\
\hline \multirow{3}{*}{ PEG J232023+081331.4 } & $g^{\prime}$ & 0.8 & \multirow{3}{*}{15} & $26.82 \pm 0.05$ & $8.60 \pm 0.35$ & $1.36 \pm 0.10$ & 28.05 & 9.46 & 0.73 & 20.62 \\
\hline & $r^{\prime}$ & 0.8 & & $26.18 \pm 0.03$ & $10.61 \pm 0.24$ & $1.81 \pm 0.12$ & 27.03 & 9.24 & 0.55 & 19.76 \\
\hline & $i^{\prime}$ & 0.9 & & $25.73 \pm 0.02$ & $9.93 \pm 0.17$ & $1.88 \pm 0.09$ & 26.53 & 8.43 & 0.53 & 19.48 \\
\hline \multirow{3}{*}{ PEG J232024+081209.0 } & $g^{\prime}$ & 0.9 & \multirow{3}{*}{14} & $26.27 \pm 0.02$ & $11.24 \pm 0.16$ & $1.63 \pm 0.08$ & 27.25 & 10.52 & 0.64 & 19.69 \\
\hline & $r^{\prime}$ & 1.0 & & $25.51 \pm 0.02$ & $10.04 \pm 0.16$ & $1.69 \pm 0.08$ & 26.44 & 9.16 & 0.59 & 19.19 \\
\hline & $i^{\prime}$ & 0.7 & & $25.64 \pm 0.03$ & $10.35 \pm 0.27$ & $1.74 \pm 0.14$ & 26.53 & 9.25 & 0.57 & 19.31 \\
\hline \multirow{3}{*}{ PEG J232037+080934.3 } & $g^{\prime}$ & 1.0 & \multirow{3}{*}{10} & $25.28 \pm 0.02$ & $5.30 \pm 0.09$ & $1.37 \pm 0.04$ & 26.51 & 5.81 & 0.73 & 20.13 \\
\hline & $r^{\prime}$ & 0.9 & & $24.80 \pm 0.02$ & $4.93 \pm 0.10$ & $1.36 \pm 0.04$ & 26.04 & 5.42 & 0.73 & 19.82 \\
\hline & $i^{\prime}$ & 0.9 & & $24.51 \pm 0.02$ & $4.89 \pm 0.10$ & $1.33 \pm 0.04$ & 25.79 & 5.52 & 0.75 & 19.51 \\
\hline \multirow{3}{*}{ PEG J232037+081336.6 } & $g^{\prime}$ & 0.8 & \multirow{3}{*}{4} & $25.55 \pm 0.03$ & $2.78 \pm 0.05$ & $1.92 \pm 0.09$ & 26.32 & 2.33 & 0.52 & 22.06 \\
\hline & $r^{\prime}$ & 0.8 & & $25.08 \pm 0.02$ & $2.98 \pm 0.03$ & $2.26 \pm 0.08$ & 25.69 & 2.30 & 0.44 & 21.48 \\
\hline & $i^{\prime}$ & 0.9 & & $24.65 \pm 0.03$ & $2.84 \pm 0.06$ & $1.62 \pm 0.09$ & 25.63 & 2.68 & 0.62 & 21.03 \\
\hline \multirow{3}{*}{ PEG J232038+081046.9 } & $g^{\prime}$ & 0.8 & \multirow{3}{*}{6} & $24.95 \pm 0.05$ & $2.77 \pm 0.10$ & $1.43 \pm 0.07$ & 26.11 & 2.91 & 0.70 & 21.20 \\
\hline & $r^{\prime}$ & 0.8 & & $24.42 \pm 0.04$ & $2.53 \pm 0.08$ & $1.25 \pm 0.05$ & 25.80 & 3.07 & 0.80 & 20.74 \\
\hline & $i^{\prime}$ & 0.9 & & $24.13 \pm 0.06$ & $2.58 \pm 0.13$ & $1.19 \pm 0.07$ & 25.60 & 3.34 & 0.84 & 20.30 \\
\hline \multirow{3}{*}{ PEG J232049+080806.2 } & $g^{\prime}$ & 0.6 & \multirow{3}{*}{6} & $25.96 \pm 0.03$ & $3.59 \pm 0.11$ & $1.26 \pm 0.07$ & 27.32 & 4.30 & 0.79 & 21.63 \\
\hline & $r^{\prime}$ & 0.6 & & $25.50 \pm 0.03$ & $3.64 \pm 0.09$ & $1.52 \pm 0.08$ & 26.57 & 3.62 & 0.66 & 21.47 \\
\hline & $i^{\prime}$ & 0.5 & & $24.94 \pm 0.03$ & $3.35 \pm 0.17$ & $1.03 \pm 0.07$ & 26.69 & 5.36 & 0.97 & 20.66 \\
\hline \multirow{3}{*}{ PEG J232054+080838.8 (int) } & $g^{\prime}$ & \multirow{3}{*}{0.0} & \multirow{3}{*}{6.5} & $24.32 \pm 0.01$ & $0.45 \pm 0.01$ & $2.11 \pm 0.11$ & 24.99 & 0.36 & 0.47 & 24.83 \\
\hline & $r^{\prime}$ & & & $23.46 \pm 0.01$ & $0.44 \pm 0.01$ & $2.18 \pm 0.10$ & 24.10 & 0.34 & 0.46 & 24.07 \\
\hline & $i^{\prime}$ & & & $22.68 \pm 0.01$ & $0.33 \pm 0.01$ & $1.89 \pm 0.06$ & 23.48 & 0.28 & 0.53 & 23.82 \\
\hline \multirow{3}{*}{ PEG J232054+080838.8 (out) } & $g^{\prime}$ & \multirow{3}{*}{0.0} & \multirow{3}{*}{6.5} & $25.13 \pm 0.01$ & $1.86 \pm 0.30$ & $0.80 \pm 0.08$ & 27.48 & 4.88 & 1.25 & 21.24 \\
\hline & $r^{\prime}$ & & & $24.89 \pm 0.01$ & $2.48 \pm 0.44$ & $0.92 \pm 0.13$ & 26.89 & 5.74 & 1.08 & 20.37 \\
\hline & $i^{\prime}$ & & & $24.50 \pm 0.02$ & $2.14 \pm 0.48$ & $0.80 \pm 0.12$ & 26.87 & 5.67 & 1.29 & 20.30 \\
\hline
\end{tabular}

Notes. $r_{\text {int }}$ and $r_{\text {ext }}$ denote the internal and external radii considered to perform the fits. In the case of the last object in the list, which displays two components in its brightness profile, a combination of two Sérsic functions was fitted to the entire profile (see text).

the two dominant galaxies, NGC 7619 and NGC 7626 (Table 2; $\langle D\rangle=50.8 \mathrm{Mpc},(m-M)=33.52 \mathrm{mag}, 1 \operatorname{arcsec}=0.248 \mathrm{kpc})$. As a reference, we also show the red sequence defined by a subsample of early-type galaxies in the Virgo cluster (Chen et al. 2010).

We can see from these plots that, in general, LSB galaxies seem to display a much wider range of colors than typical earlytype galaxies. In the particular case of the LSB objects identified in Pegasus I, with the exception of one case, they tend either to follow the red sequence of early-type galaxies or to show redder colors. Remarkably, the reddest LSB galaxies of the considered samples are found in relatively rich environments, like M 83, Leo I and Pegasus I, while their colors are much bluer than those expected for Milky Way cirrus $\left(\left(g^{\prime}-r^{\prime}\right)=1.33-2.03\right.$; Ludwig et al. 2012). In contrast, LSB galaxies found in isolation or within Hickson compact groups either follow the red sequence of early-type galaxies or display bluer colors. The LSB galaxies reported in the Virgo cluster, with the exception of one object, also behave in the same manner.

\section{2. $\left\langle\mu_{\text {eff }}\right\rangle$-luminosity diagram}

In Fig. 4 we present the $\left\langle\mu_{\text {eff }}\right\rangle$-luminosity diagram of different samples of LSB galaxies reported in the literature. As a reference, we show the location of subsamples of early-type galaxies in the Virgo cluster (Smith Castelli et al. 2013) and the NGC 5044 group (Buzzoni et al. 2012).

This plot shows that typical early-type galaxies of different luminosities tend to be placed around the locus of constant $r_{\text {eff }}=1 \mathrm{kpc}$ with a low dispersion, regardless of their environ- ment, as already reported (e.g., Smith Castelli et al. 2012, 2013, and references therein). In contrast, LSB galaxies tend to display a wider range in $r_{\text {eff }}$, spanning from LG dSph galaxies (the smallest LSB galaxies detected so far) to the so-called UDGs in more distant groups and clusters (which are the largest examples of LSB galaxies). In particular, the LSB galaxies detected in Pegasus I seem to show $r_{\text {eff }}$ similar to those of early-type galaxies, filling the gap between the smallest and the largest LSB systems.

In this plot, the references to the different samples are ordered according to their reported distances (or those of the environments towards which they were detected), increasing from top to bottom. A distance-size trend is apparent; that is, the smallest objects are identified in the Local Group, while the largest systems reported in the literature are detected in more distant groups/clusters.

To quantify this appreciation, Fig. 5 shows the mean, minimum, and maximum (absolute and apparent) $r_{\text {eff }}$ of different samples. All the panels show the same trend: while the largest objects are found in the most distant environments, the smallest ones are only detected in the Local Volume.

\subsection{Andromeda's satellites}

Regarding the likely presence of a bias in the detection of LSB galaxies, we wonder which would be the appearance of, for example, the M 31 (Andromeda) dSph satellites at the distance of Pegasus I. Figure 6 shows models of six of these galaxies obtained from the brightness profiles reported by Caldwell et al. (1992) and Caldwell (1999), overimposed on one of our 
\ M83 LSB galaxies (Müller et al. 2015)

$\diamond$ Leo I dSphs (Müller et al. 2018)

- NGC 7331 dSphs (Ludwig et al. 2012)

Virgo early-type galaxies (Chen et al. 2010)

V Virgo LSB galaxies (Prole et al. 2018)

Fornax LSB galaxies (Venhola et al. 2017)

- NGC 5485 UDGs (Merritt et al. 2017)

* HCG 44 LSB galaxies (Smith Castelli et al. 2016)

- Isolated dSphs (Makarov et al. 2015)

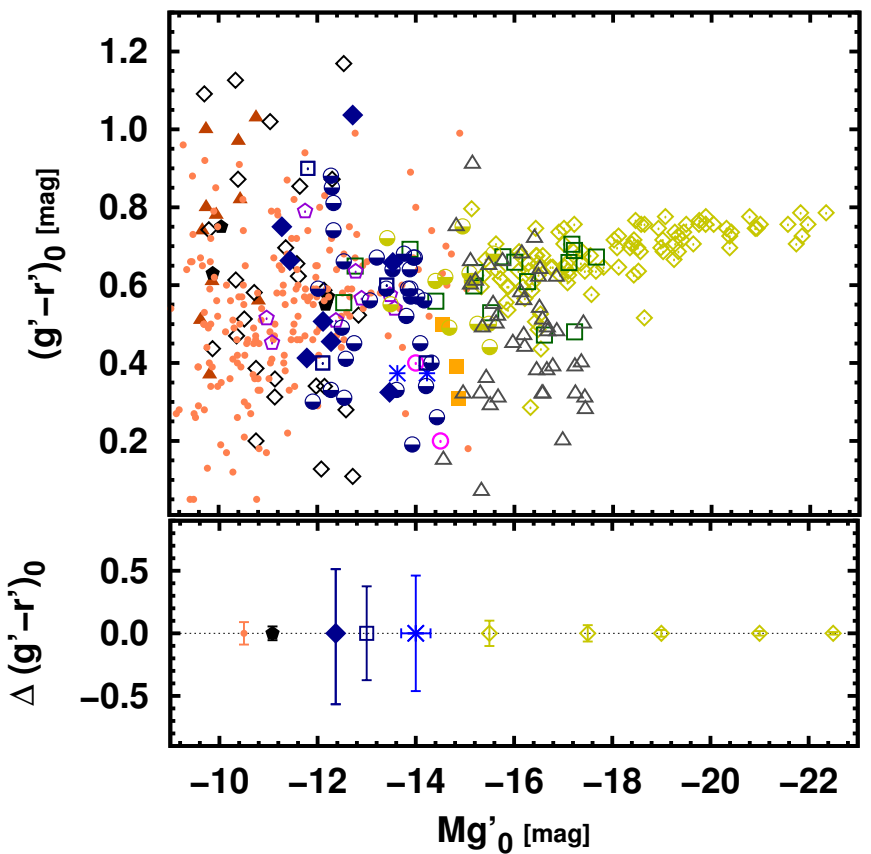

$\odot$ Isolated LSB galaxies (Greco et al. 2018)

$\square$ NGC 5044 dE/dSO (Buzzoni et al. 2012)

$\checkmark$ NGC 5044 dSphs (Buzzoni et al. 2012)

- Pegasus I LSB galaxies (Shi et al. 2017)

Pegasus I UDGs (Shi et al. 2017)

- Pegasus I LSB galaxies (this work)

$\nabla$ HCG 07/25/98 UDGs (Román et al. 2017)

$\triangle$ Pegasus II UDGs (Shi et al. 2017)

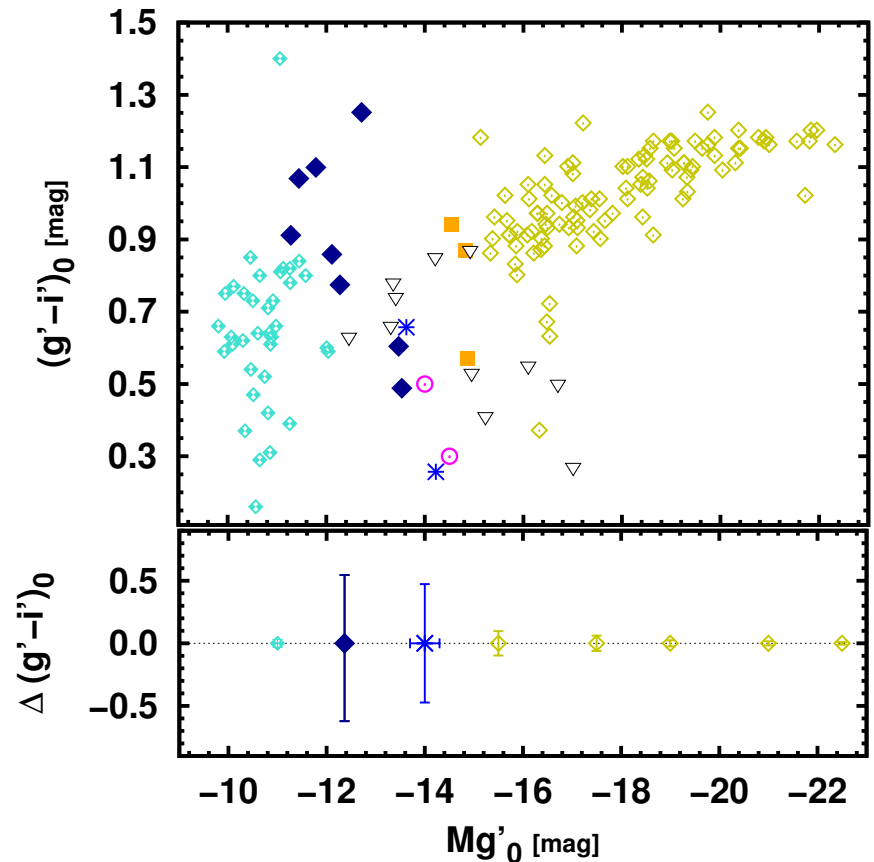

Fig. 3. Color-magnitude diagrams showing the location of the LSB galaxies presented in this work, assuming that they are at the distance of Pegasus I (blue filled diamonds). As a reference, we plot the red sequence defined by a subsample of early-type galaxies in the central region of the Virgo cluster (green open diamonds; Chen et al. 2010), and a sample of dE/dS0 galaxies in the NGC 5044 group (open squares without a dot; Buzzoni et al. 2012). We also include different samples of LSB, dwarf spheroidal (dSph), and ultra-diffuse galaxies (UDGs) reported in the literature. They are listed in the top margin of the plot, according to their distances or those of the environments towards which they were detected, in ascending order from top to bottom, and from left to right. They are: M 83 LSB galaxies (Müller et al. 2015), Leo I dSphs (Müller et al. 2015), NGC 7331 dSphs (Ludwig et al. 2012), Virgo LSB galaxies (Mihos et al. 2015; Prole et al. 2018), Fornax LSB galaxies (Venhola et al. 2017), NGC 5485 UDGs (Shi et al. 2017), HCG 44 LSB galaxies (Smith Castelli et al. 2016), Isolated dSphs (Makarov et al. 2015), Isolated LSB galaxies (Greco et al. 2018), NGC 5044 dSphs (Buzzoni et al. 2012), Pegasus I LSB/UDGs (Shi et al. 2017), HCG 07, HCG 25 and HCG 98 (Román \& Trujillo 2017b) and Pegasus II UDGs (Shi et al. 2017). In both diagrams, the mean error bars of the different samples, when available, are shown in the panels below.

Gemini-GMOS fields. In addition, in Fig. 7 we compare the surface-brightness profiles of our LSB galaxies with those of the Andromeda satellites scaled to the distance of Pegasus I. From these figures it can be seen that Andromeda's dSphs would display $2<r_{\text {tot }}<8$ arcsec, and $24<\mu_{0 g}<26 \mathrm{mag} \mathrm{arcsec}^{-2}$. Their central surface brightnesses would thus be relatively low, but still brighter than those of the LSB galaxies detected in Pegasus I; however, their apparent sizes would be much smaller in comparison with the LSB galaxies in our sample.

Therefore, it is evident that we have certainly missed any putative objects similar to the dSph satellites of Andromeda in our visual selection of extended and diffuse galaxies. In a following paper we will present the analysis of our images regarding the detection of counterparts of Andromeda's dSph galaxies towards Pegasus I (González et al., in prep.).

\subsection{Sérsic index}

From Fig. 8 we can see that LSB galaxies display Sérsic indices in a relatively narrow range $(n \lesssim 2)$ in comparison to early-type galaxies. The objects detected in the direction of Pegasus I (except one galaxy) have brightness profiles that can be fitted by a single Sérsic model with $0.44<n<0.97$. The remaining galaxy presents two components: a nucleus with $\left(g^{\prime}-i^{\prime}\right)=1.01 \mathrm{mag}$, marginally resolved in the $g^{\prime}, r^{\prime}$ and $i^{\prime}$ images, and an external component with $1.08<n<1.29$, depending on the filter (see Table 4). It is important to note that the Sérsic index of the external component is within the expected range for LSB galaxies (e.g., Mihos et al. 2015; Venhola et al. 2017). Although some nucleated LSB galaxies have been identified towards nearby groups such as M 101 (Bennet et al. 2017) and Leo I (Müller et al. 2018), as far as we know the role of nuclei in connection with the formation and evolution of LSB galaxies has not been studied in depth.

\section{Discussion and conclusions}

In this work we present eight LSB galaxies detected in the central region of the Pegasus I group. Considering their photometric and structural properties, these galaxies show similar 


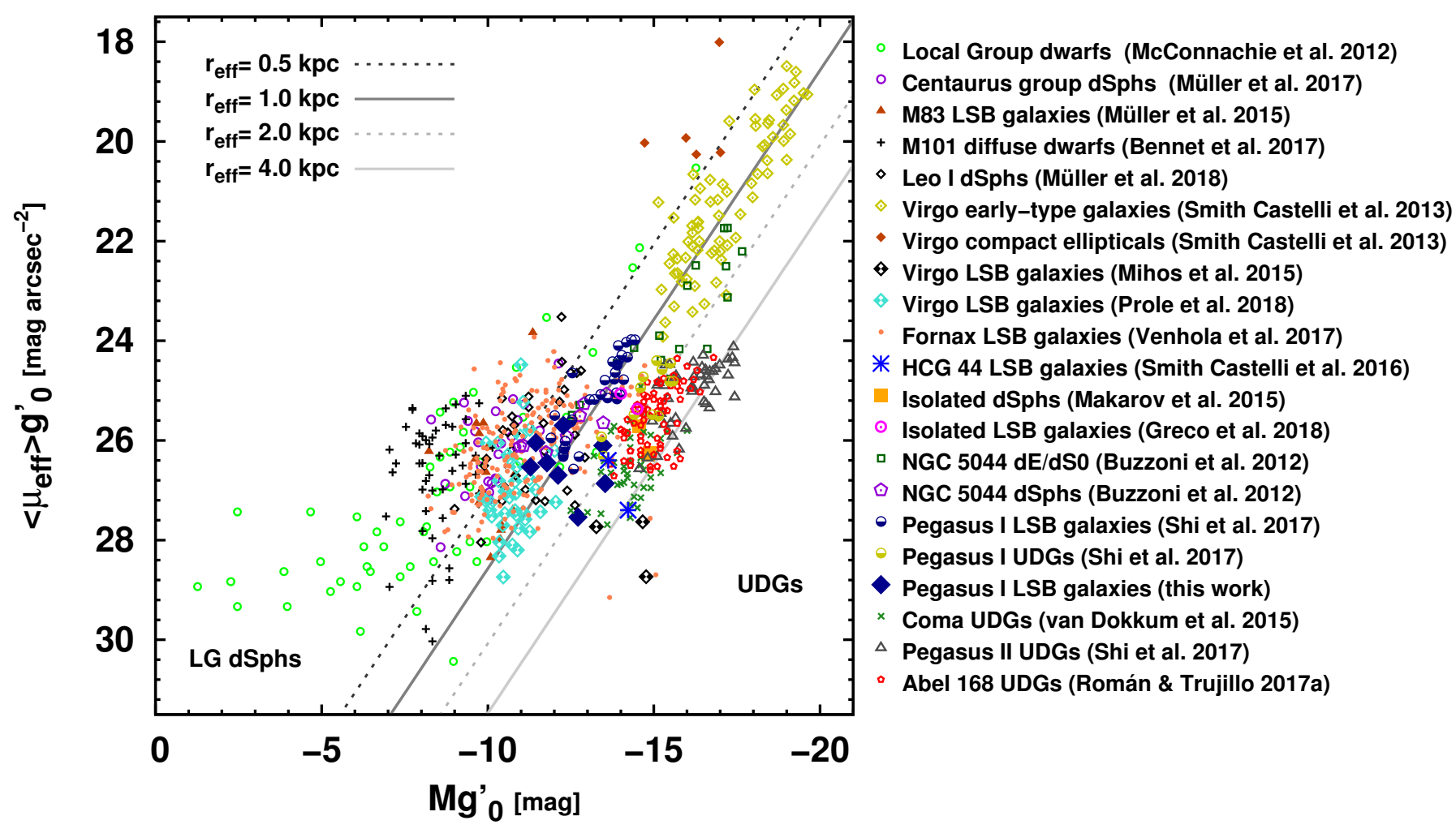

Fig. 4. $\left\langle\mu_{\text {eff }}\right\rangle$-luminosity diagram of the early-type galaxies in the central region of the Virgo cluster (Smith Castelli et al. 2013), showing the location of the LSB galaxies presented in this work, assuming they are at the distance of Pegasus I. For comparison, we also include different samples of LSB galaxies, dSph galaxies, and UDGs reported in the literature. They are listed in the right margin of the plot according to their distances or those of the environments towards which they were detected, in ascending order from top to bottom. They are: Local Group dwarfs (McConnachie 2012), Centaurus dSphs (Müller et al. 2017), M 83 LSB galaxies (Müller et al. 2015), M 101 dwarfs (Bennet et al. 2017), Leo I dSphs (Müller et al. 2015), Virgo LSB galaxies (Mihos et al. 2015; Prole et al. 2018), Fornax LSB galaxies (Venhola et al. 2017), HCG 44 LSB galaxies (Smith Castelli et al. 2016), Isolated dSphs (Makarov et al. 2015), Isolated LSB galaxies (Greco et al. 2018), NGC 5044 dE/dS0 and dSph galaxies (Buzzoni et al. 2012), Pegasus I LSB/UDGs (Shi et al. 2017), Coma UDGs (van Dokkum et al. 2015), Pegasus II UDGs (Shi et al. 2017) and Abell 168 UDGs (Román \& Trujillo 2017a). The lines of constant $r_{\text {eff }}$ are only valid for exponential profiles.

characteristics to those of LSB galaxies reported in the literature in other environments. In particular, we found that three of these galaxies can be classified as UDGs, following the criteria established by van Dokkum et al. (2015): $\mu_{0, g^{\prime}} \gtrsim 24 \mathrm{mag} \operatorname{arcsec}^{-2}$ and $r_{\text {eff }} \gtrsim 1.5 \mathrm{kpc}$. It is important to note that these criteria do not imply that UDGs are distinct from the general galaxy population; they are simply large and extremely diffuse objects. Two of these UDGs (PEG J232023+081331.4 and PEG J232024+081209.0) are located within Field 4 (Fig. 1), at a projected distance of less than 2 arcmin from NGC 7619. The third one (PEG J232037+080934.3) is placed in Field 7, at a projected distance of $\sim 3$ arcmin from NGC 7626. The rest of the detected LSBs do not present any distinctive peculiarities, except PEG J232054+080838.8 (located in Field 8 at a projected distance of $\sim 5$ arcmin from NGC 7626), which displays a nucleus.

Despite many efforts to understand the formation of UDGs, the observations have so far led to diverse scenarios. For example, van Dokkum et al. (2015) suggested that some UDGs could be "failed" galaxies, which lost their gas after the formation of the first generation of stars, being strongly dominated by dark matter. This scenario seems to be in agreement with the reported velocity dispersion of the UDG Dragonfly 44 in the Coma cluster (van Dokkum et al. 2016). Similarly, the evidence of a high number of globular clusters in the UDG Dragonfly 17 supports the idea that it could be a failed galaxy with a halo mass similar to the Large Magellanic Cloud (Beasley \& Trujillo 2016).
From a theoretical point of view, Amorisco \& Loeb (2016) claimed that UDGs can be easily explained by a standard model of disk formation. They suggested that UDGs are part of the dwarf galaxy population with a particularly high original angular momentum. In this scenario, the high angular momentum makes the UDGs more flat and extended than typical dEs. Similarly, through Millennium and Phoenix simulations of large clusters, Rong et al. (2017) showed that UDGs are genuine dwarf galaxies that can naturally emerge from the $\Lambda$ CDM model. This model accurately reproduces the observed properties of UDGs in the nearby clusters. On the other hand, the existence of UDGs in the field has also been theoretically predicted by Di Cintio et al. (2017), using cosmological simulations from the Numerical Investigation of a Hundred Astrophysical Objects (NIHAO) project. They showed that UDGs naturally form in dwarf-sized halos due to episodes of gas outflows associated with star formation. More recently, Baushev (2018) suggested that a possible mechanism for the formation of UDGs could be the central collision of galaxies in their youth stage. This collision would heat the gas of the system, expelling it from the galaxies but without affecting their dark matter and stellar components, leaving them with the typical observational properties for this kind of object.

Initially, rich clusters were considered the natural place to find UDGs. Some of the studies that supported this idea focused on Fornax (Muñoz et al. 2015), Coma (Koda et al. 2015; 

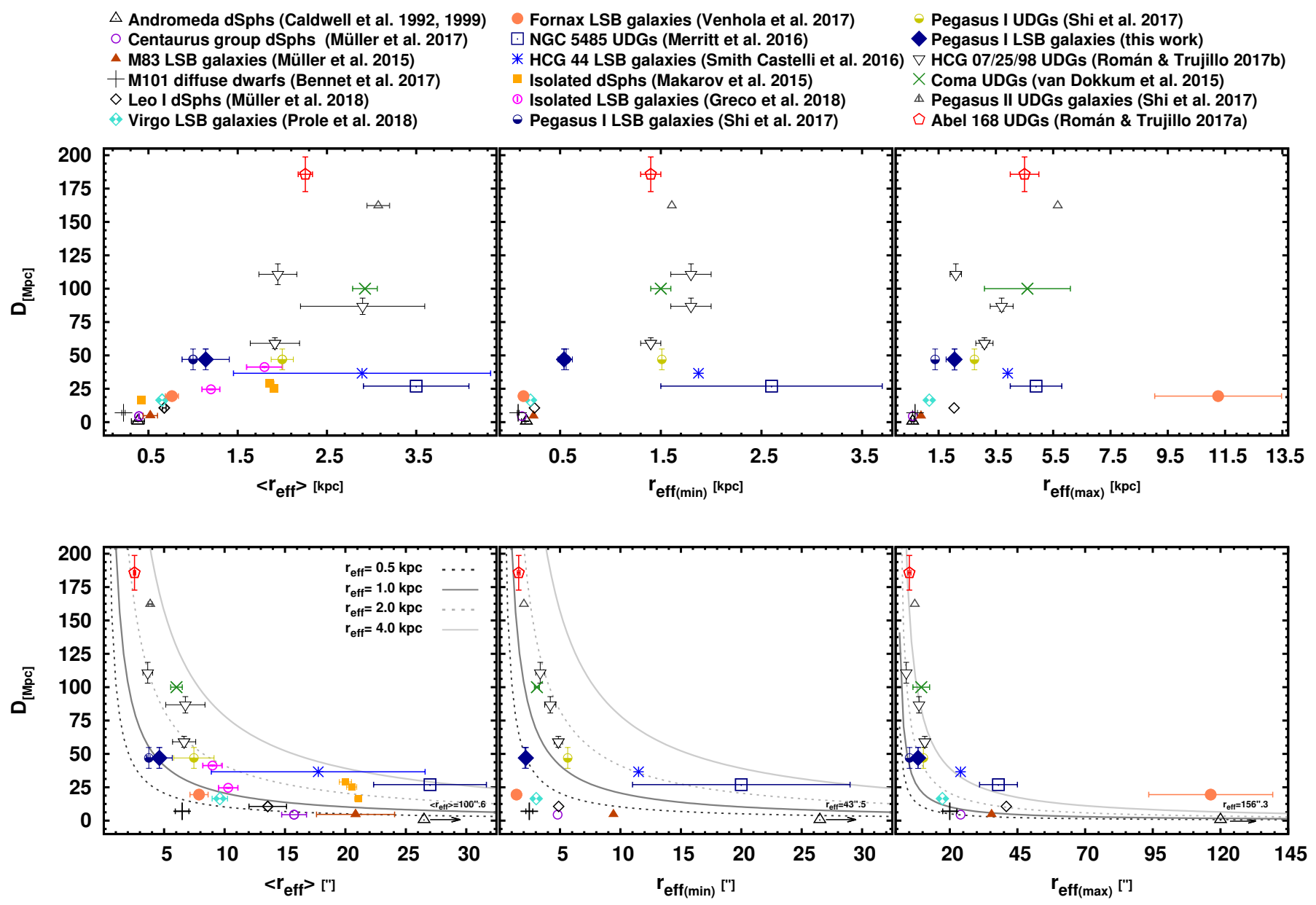

Fig. 5. Mean (left panels), minimum (middle panels), and maximum (right panels) absolute (top panels) and apparent (bottom panels) effective radius of several samples of LSB galaxies vs. the distances to the environments towards which they were detected.

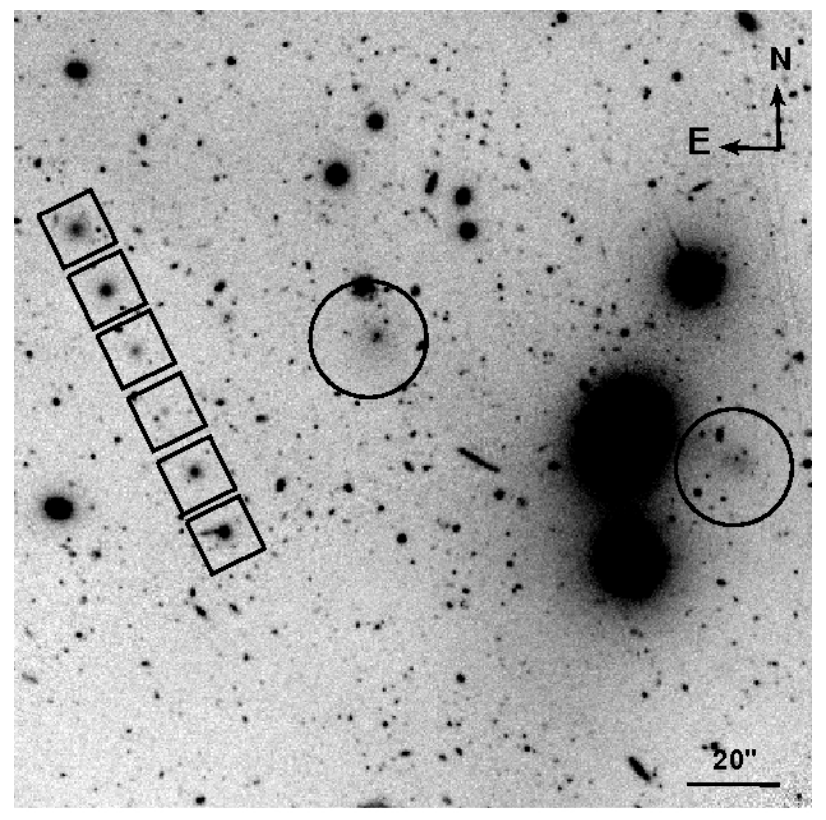

Fig. 6. $2.85 \times 2.85$ arcmin frame in the $g^{\prime}$ filter showing two of the LSB galaxies detected in Pegasus I (circles). In order to compare the appearance that dSph Andromeda's satellites would have at the distance of Pegasus I, we added to this image the models obtained from their brightness profiles (Caldwell et al. 1992; Caldwell 1999) scaled to that distance (squares). van Dokkum et al. 2015) and Virgo (Mihos et al. 2015; Davies et al. 2016). More distant groups in which UDGs have been reported are Pegasus II (Shi et al. 2017) and Abell 168 (Román \& Trujillo 2017a). However, there is recent evidence for the existence of such extended objects outside rich clusters. For example, additional UDGs have been detected in HCG 07, HCG 25, and HCG 98 (Román \& Trujillo 2017b); M 101 (Merritt etal. 2016); and HCG 44 (Smith Castelli et al. 2016). Moreover, this kind of object has also been reported in the field (Makarov et al.2015; Greco et al. 2018), underlining the fact that UDGs can be found in diverse environments.

Following van der Burg et al. (2016), UDGs should not survive near the center of clusters, where tidal forces due to the cluster mass would prevent their formation. At odds with this statement, we have found three UDG candidates well inside the central region of Pegasus I, very close - in projection - to massive elliptical galaxies. However, it is worth noting the results found in Abell 168 (Román \& Trujillo 2017a) and the three isolated groups HCG 07/25/98 (Román \& Trujillo 2017b), where the structural properties of UDGs change towards the center of the groups showing a decrease in $r_{\text {eff }}$, fainter $\left\langle\mu_{\text {eff }}\right\rangle$, and larger values of the Sérsic index $n$. In our case, the most central UDGs display larger $r_{\mathrm{eff}}$, fainter $\left\langle\mu_{\mathrm{eff}}\right\rangle$, and lower $n$ values (in the $r^{\prime}$ and $i^{\prime}$ filters) than the more distant one. However, this evidence is based on only three objects, and it is thus not conclusive. In addition, from Fig. 4, it can be seen that LSB galaxies reported by Shi et al. (2017) in more external regions of 


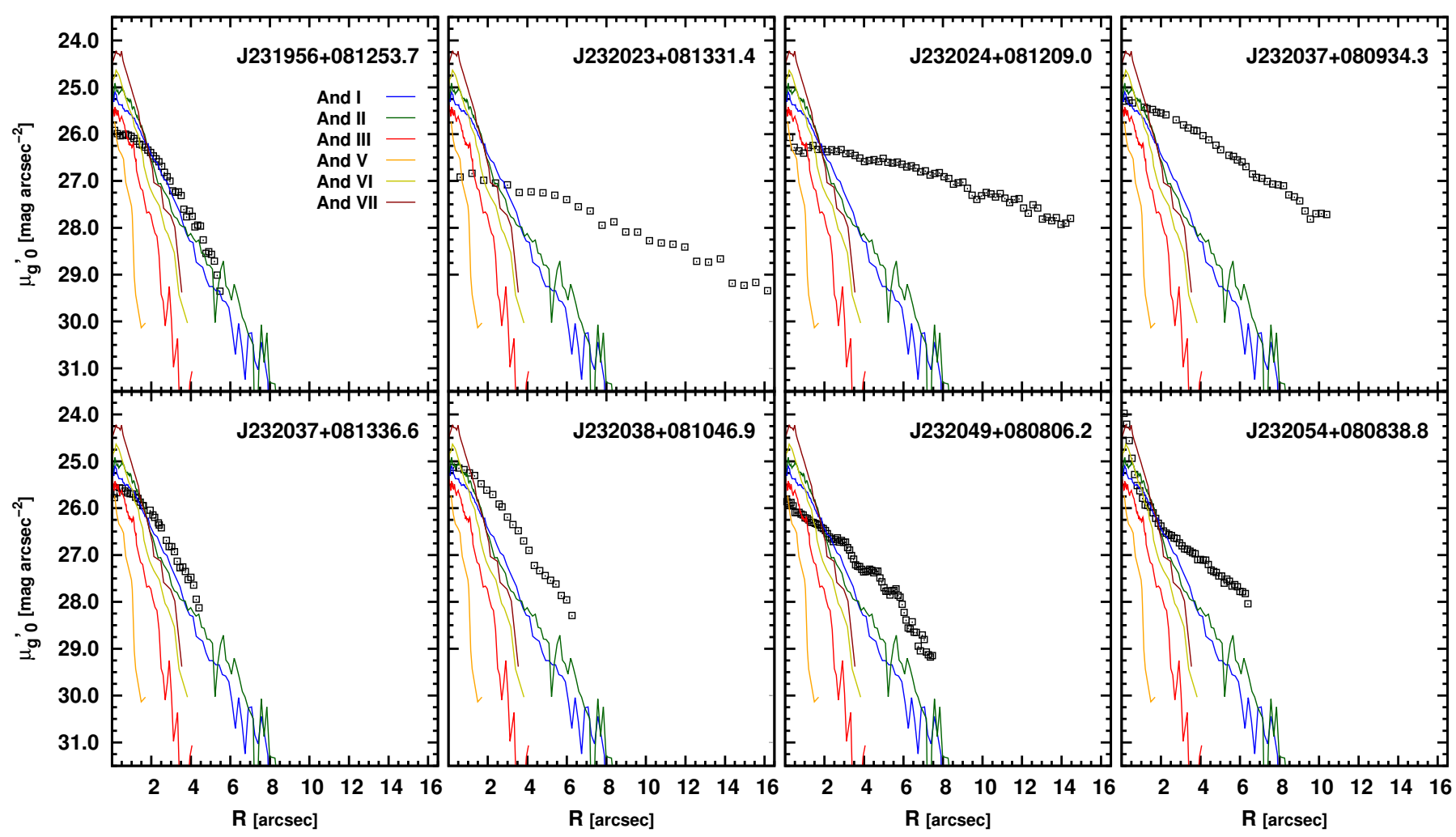

Fig. 7. Comparison between the $g^{\prime}$ brightness profiles of the LSB galaxies detected in Pegasus I (black open squares with a dot) and those of six M 31 dSph satellites (Caldwell et al. 1992; Caldwell 1999). Andromeda's satellites' profiles were transformed from the $V$-band to the $g^{\prime}$-band through the relations presented by Fukugita et al. (1995), and scaled to the distance of Pegasus I.

Pegasus I than those observed by us display similar $r_{\text {eff }}$ than those of the central regions, but they show brighter $\left\langle\mu_{\mathrm{eff}}\right\rangle$ (these authors do not provide $n$ values for these galaxies). Considering all the UDGs in Pegasus I reported so far, it is found that they spread over a wide range of colors both in $\left(g^{\prime}-i^{\prime}\right)$ and $\left(g^{\prime}-r^{\prime}\right)$, covering both the red and the blue sequences. No correlation is found among UDGs between color and the environment where they reside (Román \& Trujillo 2017a; Shi et al. 2017).

In general, LSB galaxies show smooth surface brightness profiles that are well characterized by single-component Sérsic functions, with index $n \lesssim 2$. In particular, the galaxies detected in the direction of Pegasus I display a more restricted range of values $(0.44<n<0.97)$. It is interesting to note that, while typical early-type galaxies follow a well-defined luminosity- $n$ relation (Caon et al. 1993; Cellone et al. 1994), the LSB galaxies, in contrast, display $n$ values scattering around $n \lesssim 1$, showing no trend with luminosity. There is an opposite situation if we consider the color-magnitude or the $\left\langle\mu_{\text {eff }}\right\rangle$-luminosity planes, where typical early-type galaxies display well-delineated sequences while LSB galaxies show no clear trend at all. In particular, high- $n$ profiles are usually explained by major mergers and violent relaxation or by numerous minor mergers, while low- $n$ profiles are usually associated with secular evolution (Elmegreen et al. 2008, and references therein). In this context, and given the remarkably different behavior between the $n$ values displayed by typical early-type galaxies and LSB galaxies (regardless of their sizes), it can be proposed that both types of system arise from different formation processes, which also implies different formation timescales (rapid and slow, respectively). Two distinct formation pathways might also explain the different behaviors observed between other (color-chemical,

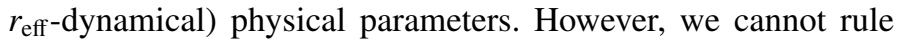
out that some of these objects may have a tidal origin. In the cases of PEG J232023+081331.4, PEG J232024+081209.0, and PEGJ232049+080806.3, we are able to identify some extended and very diffuse substructures in their images that might be associated with this kind of process (see Figs. A.1, A.2, and A.6). We note that one of these structures presents $\mu_{r^{\prime}} \gtrsim 27$ mag $\operatorname{arcsec}^{-2}$.

Another point to note is the correlation that seems to exist between the effective radii of LSB galaxies and the distances of their host clusters/groups, in the sense that the actual sizes of the LSB galaxies increase with distance. This correlation could simply be a selection effect due to the limitations we have to detect such low-surface-brightness objects at large distances. Small distant LSB galaxies can be confused with background objects, while large nearby LSB galaxies might not be detected because of their exceedingly large angular sizes (although the probability of finding large LSB galaxies in the a small volume around the Local Group must be relatively low). Recently, Müller et al. (2018) found possible extremely large UDGs in the Leo I group $(D \sim 10.7 \mathrm{Mpc})$. If these UDGs are confirmed to be LeoI members, they would be some of the closest.

Within this context, where the formation scenarios and evolutionary paths followed by these objects remain speculative, the database of observed LSB galaxies in different environments should be enlarged, thus providing the basis to better constrain models of LSB formation and evolution. As a future work, we expect to obtain spectroscopic data of LSB galaxies to confirm their membership to Pegasus I. This will certainly help us to unveil the real nature of these extreme stellar systems. 

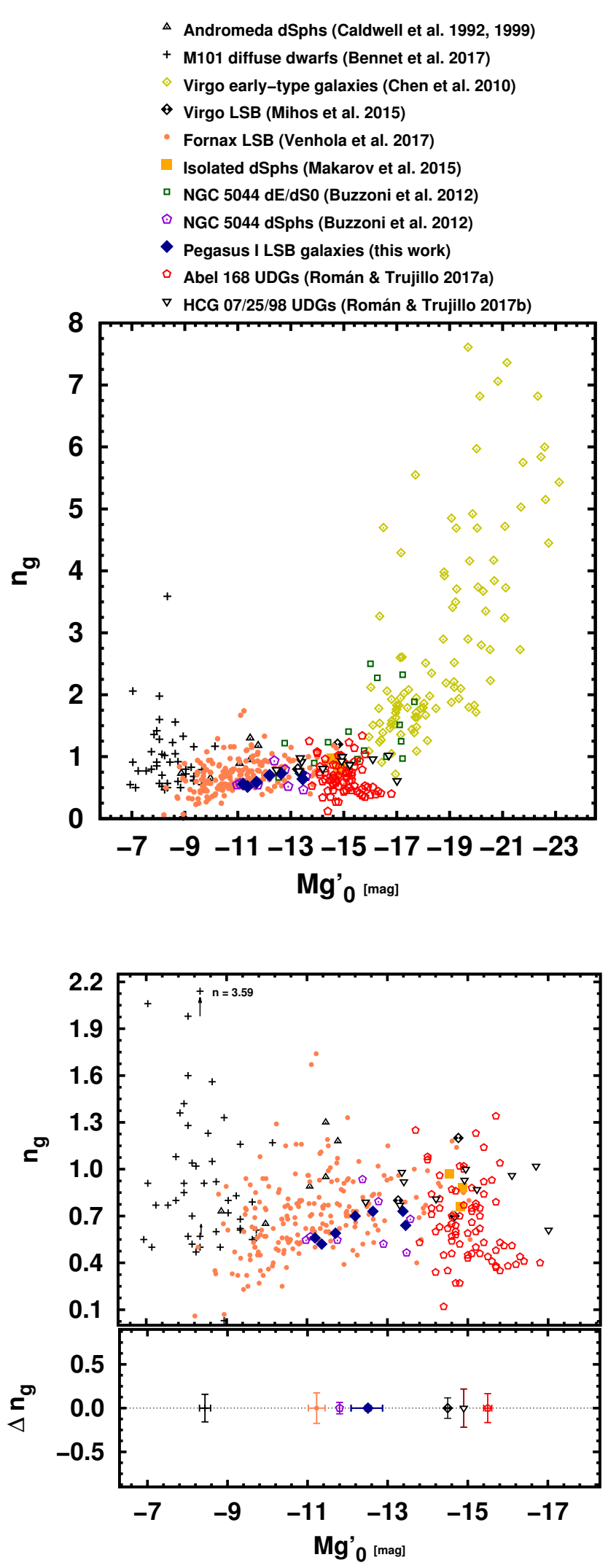

Fig. 8. Sérsic index vs. luminosity. Upper panel: we show the location of different samples of LSB galaxies and, as a reference, that of subsamples of early-type galaxies of Virgo and NGC 5044. LSB galaxies show a very narrow range of $n$ values in comparison to those shown by typical early-type galaxies. Lower panel: only the LSB galaxy samples are shown, to allow for a more detailed display. In both panels, symbol code is as given in the top margin of the upper panel.
Acknowledgements. Based on observations obtained at the Gemini Observatory (Program GN-2008B-Q-14, GN-2012A-Q-55, GN-2014A-Q-70, GN-2014B-Q17 and GN-2015B-Q-13), which is operated by the Association of Universities for Research in Astronomy, Inc., under a cooperative agreement with the NSF on behalf of the Gemini partnership: the National Science Foundation (United States), the National Research Council (Canada), CONICYT (Chile), Ministério da Ciência, Tecnologia e Inovação (Brazil) and Ministerio de Ciencia, Tecnología e Innovación Productiva (Argentina). This research has made use of the NASA/IPAC Extragalactic Database (NED) which is operated by the Jet Propulsion Laboratory, California Institute of Technology, under contract with the National Aeronautics and Space Administration. Funding for SDSS-III has been provided by the Alfred P. Sloan Foundation, the Participating Institutions, the National Science Foundation, and the US Department of Energy Office of Science. The SDSS-III web site is http: //www . sdss3 . org/. SDSS-III is managed by the Astrophysical Research Consortium for the Participating Institutions of the SDSS-III Collaboration including the University of Arizona, the Brazilian Participation Group, Brookhaven National Laboratory, Carnegie Mellon University, University of Florida, the French Participation Group, the German Participation Group, Harvard University, the Instituto de Astrofisica de Canarias, the Michigan State/Notre Dame/JINA Participation Group, Johns Hopkins University, Lawrence Berkeley National Laboratory, Max Planck Institute for Astrophysics, Max Planck Institute for Extraterrestrial Physics, New Mexico State University, New York University, Ohio State University, Pennsylvania State University, University of Portsmouth, Princeton University, the Spanish Participation Group, University of Tokyo, University of Utah, Vanderbilt University, University of Virginia, University of Washington, and Yale University. This work was funded with grants from Consejo Nacional de Investigaciones Científicas y Técnicas and Universidad Nacional de La Plata (Argentina).

\section{References}

Aaronson, M., Mould, J., Huchra, J., et al. 1980, ApJ, 239, 12

Amorisco, N. C., \& Loeb, A. 2016, MNRAS, 459, L51

Balcells, M., \& Carter, D. 1993, A\&A, 279, 376

Baushev, A. N. 2018, New Astron., 60, 69

Beasley, M. A., \& Trujillo, I. 2016, ApJ, 830, 23

Bennet, P., Sand, D. J., Crnojević, D., et al. 2017, ApJ, 850, 109

Bertin, E., \& Arnouts, S. 1996, A\&AS, 117, 393

Birkinshaw, M., \& Davies, R. L. 1985, ApJ, 291, 32

Buzzoni, A., Cellone, S. A., Saracco, P., \& Zucca, E. 2012, MNRAS, 420, 3427 Caldwell, N. 1999, AJ, 118, 1230

Caldwell, N., Armandroff, T. E., Seitzer, P., \& Da Costa, G. S. 1992, AJ, 103, 840

Canizares, C. R., Donahue, M. E., Trinchieri, G., Stewart, G. C., \& McGlynn, T. A. 1986, ApJ, 304, 312

Caon, N., Capaccioli, M., \& D’Onofrio, M. 1993, MNRAS, 265, 1013

Cellone, S. A., Forte, J. C., \& Geisler, D. 1994, ApJS, 93, 397

Chen, C.-W., Côté, P., West, A. A., Peng, E. W., \& Ferrarese, L. 2010, ApJS, 191, 1

Chincarini, G., \& Rood, H. J. 1976, PASP, 88, 388

Dabringhausen, J., \& Kroupa, P. 2013, MNRAS, 429, 1858

Davies, L. J. M., Robotham, A. S. G., Driver, S. P., et al. 2016, MNRAS, 455, 4013

de Vaucouleurs, G., de Vaucouleurs, A., Corwin, Jr., H. G., et al. 1992, VizieR Online Data Catalog: VII/137

Di Cintio, A., Brook, C. B., Dutton, A. A., et al. 2017, MNRAS, 466, L1

Elmegreen, B. G., Bournaud, F., \& Elmegreen, D. M. 2008, ApJ, 688, 67

Fujita, Y. 2003, PASJ, 55, 593

Fukugita, M., Shimasaku, K., \& Ichikawa, T. 1995, PASP, 107, 945

Graham, A. W., \& Worley, C. C. 2008, MNRAS, 388, 1708

Greco, J. P., Goulding, A. D., Greene, J. E., et al. 2018, ApJ, 866, 112

Impey, C., \& Bothun, G. 1997, ARA\&A, 35, 267

Jedrzejewski, R. I. 1987, MNRAS, 226, 747

Jedrzejewski, R., \& Schechter, P. L. 1988, ApJ, 330, L87

Kim, D.-W., Kim, E., Fabbiano, G., \& Trinchieri, G. 2008, ApJ, 688, 931

Koda, J., Yagi, M., Yamanoi, H., \& Komiyama, Y. 2015, ApJ, 807, L2

Levy, L., Rose, J. A., van Gorkom, J. H., \& Chaboyer, B. 2007, AJ, 133, 1104

Ludwig, J., Pasquali, A., Grebel, E. K., \& Gallagher, III., J. S. 2012, AJ, 144, 190

MacArthur, L. A., Courteau, S., \& Holtzman, J. A. 2003, ApJ, 582, 689

Makarov, D. I., Sharina, M. E., Karachentseva, V. E., \& Karachentsev, I. D. 2015, A\&A, 581, A82

McConnachie, A. W. 2012, AJ, 144, 4

Merritt, A., van Dokkum, P., Danieli, S., et al. 2016, ApJ, 833, 168

Mihos, J. C., Durrell, P. R., Ferrarese, L., et al. 2015, ApJ, 809, L21

Müller, O., Jerjen, H., \& Binggeli, B. 2015, A\&A, 583, A79

Müller, O., Jerjen, H., \& Binggeli, B. 2017, A\&A, 597, A7 
Müller, O., Jerjen, H., \& Binggeli, B. 2018, A\&A, 615, A105

Muñoz, R. P., Eigenthaler, P., Puzia, T. H., et al. 2015, ApJ, 813, L15

O’Neil, K., Bothun, G. D., Schombert, J., Cornell, M. E., \& Impey, C. D. 1997, AJ, 114, 2448

Prole, D. J., Davies, J. I., Keenan, O. C., \& Davies, L. J. M. 2018, MNRAS, 478, 667

Ramella, M., Geller, M. J., Pisani, A., \& da Costa, L. N. 2002, AJ, 123, 2976

Randall, S. W., Jones, C., Kraft, R., Forman, W. R., \& O'Sullivan, E. 2009, ApJ, 696, 1431

Richter, O.-G., \& Huchtmeier, W. K. 1982, A\&A, 109, 155

Román, J., \& Trujillo, I. 2017a, MNRAS, 468, 703

Román, J., \& Trujillo, I. 2017b, MNRAS, 468, 4039

Rong, Y., Guo, Q., Gao, L., et al. 2017, MNRAS, 470, 4231

Sérsic, J. L. 1963, Bol. Asoc. Argent. Astron. La Plata Argent., 6, 41

Shi, D. D., Zheng, X. Z., Zhao, H. B., et al. 2017, ApJ, 846, 26

Smith Castelli, A. V., Cellone, S. A., Faifer, F. R., et al. 2012, MNRAS, 419, 2472
Smith Castelli, A. V., González, N. M., Faifer, F. R., \& Forte, J. C. 2013, ApJ, 772, 68

Smith Castelli, A. V., Faifer, F. R., \& Escudero, C. G. 2016, A\&A, 596, A23

Tody, D. 1986, in Instrumentation in astronomy VI, ed. D. L. Crawford, Proc. SPIE, 627, 733

Tonry, J. L., Dressler, A., Blakeslee, J. P., et al. 2001, ApJ, 546, 681 Trentham, N. 1997, MNRAS, 286, 133

Trinchieri, G., Fabbiano, G., \& Kim, D.-W. 1997, A\&A, 318, 361

Tully, R. B., Courtois, H. M., Dolphin, A. E., et al. 2013, AJ, 146, 86

Vader, J. P., \& Chaboyer, B. 1994, AJ, 108, 1209

van der Burg, R. F. J., Muzzin, A., \& Hoekstra, H. 2016, A\&A, 590, A20

van Dokkum, P. G., Abraham, R., Merritt, A., et al. 2015, ApJ, 798, L45

van Dokkum, P., Abraham, R., Brodie, J., et al. 2016, ApJ, 828, L6

Venhola, A., Peletier, R., Laurikainen, E., et al. 2017, A\&A, 608, A142

Vigroux, L., Boulade, O., \& Rose, J. A. 1989, AJ, 98, 2044

Zwicky, F. 1942, PASP, 54, 185

Zwicky, F., Karpowicz, M., \& Kowal, C.T. 1965, Catalogue of Galaxies and of Clusters of Galaxies, 5 


\section{Appendix A: LSB galaxies identified in Pegasus I}

In this section we present similar figures to Fig. 2, for the rest of the extended LSB galaxies identified in Pegasus I.
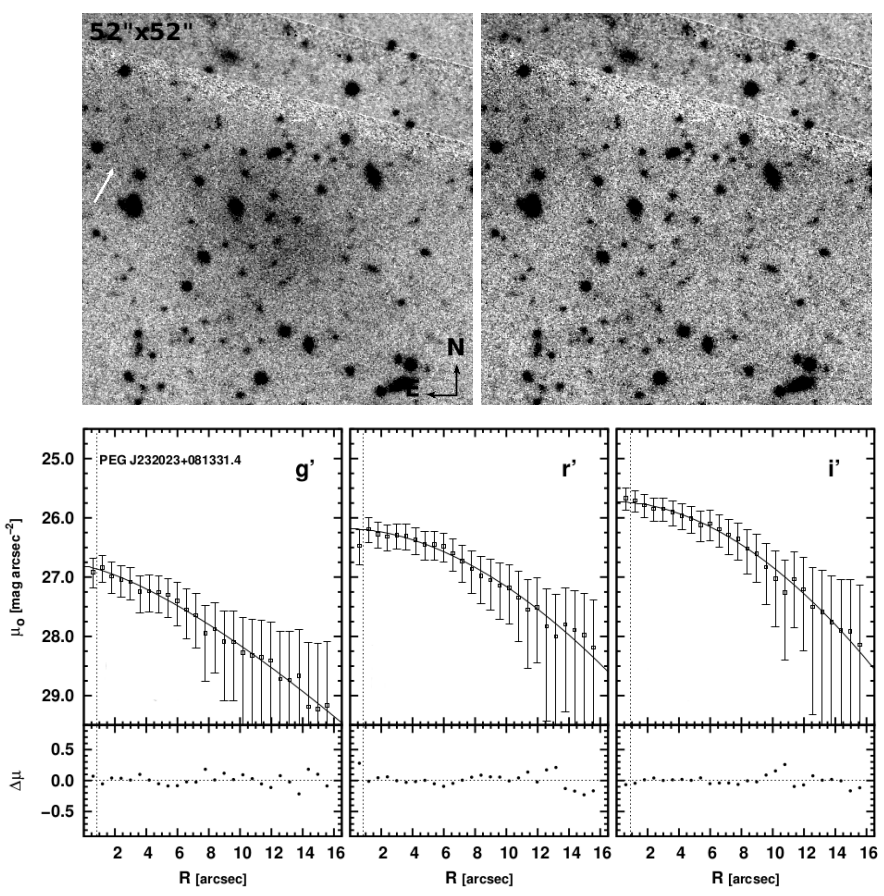

Fig. A.1. As in Fig. 2 but for galaxy PEG J232023+081331.4. In this case, the size of the frames in the upper panels is $52 \times 52 \mathrm{arcsec}$. There seems to be an extremely faint, low-surface-brightness structure connected to the galaxy (white arrow). This structure might be evidence of a tidal origin for the galaxy, or of an interaction with a tidal structure. This tidal structure may be affecting the galaxy's colors. The galaxy is superposed on the dithering pattern of the gaps between the CCDs, resulting in larger photometric errors.
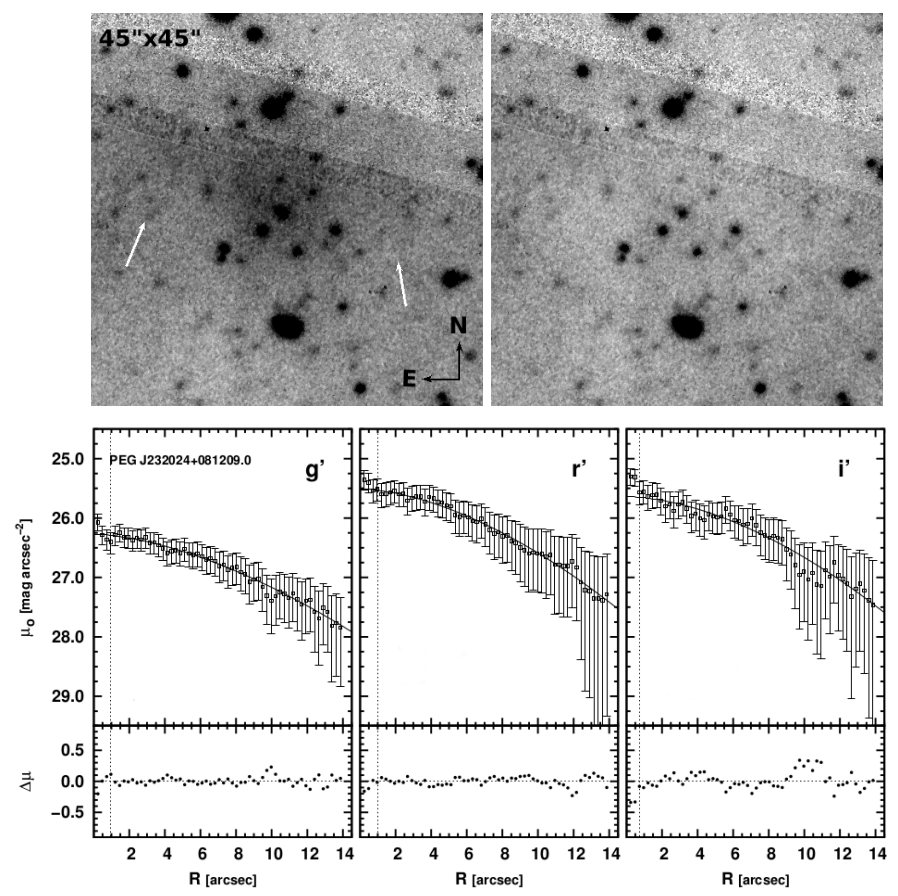

Fig. A.2. As in Fig. 2 but for galaxy PEG J232024+081209.0. In this case, the size of the frames in the upper panels is $45 \times 45$ arcsec. an extremely faint low-surface-brightness structure connected to the galaxy (white arrows). This structure might be evidence of a tidal origin for the galaxy, or of an interaction with a tidal structure. This tidal structure may be affecting the galaxy's colors. The galaxy is superposed on the dithering pattern of the gaps between the CCDs, resulting in relatively large photometric errors.
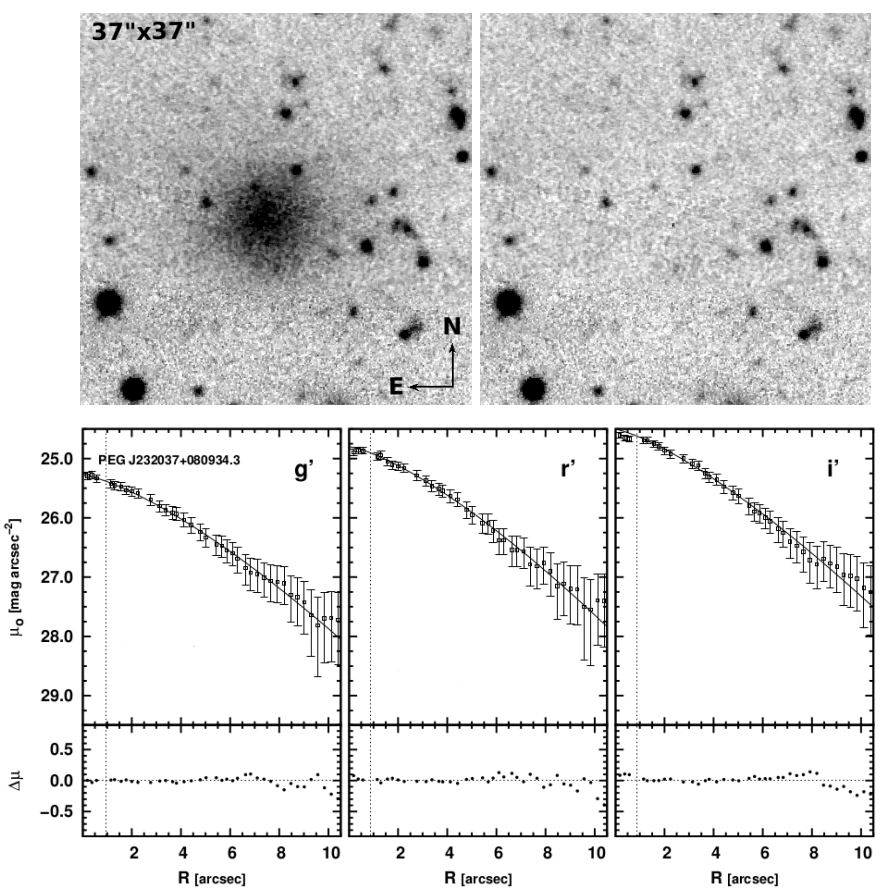

Fig. A.3. As in Fig. 2 but for galaxy PEG J232037+080934.3. In this case, the size of the frames in the upper panels is $37 \times 37$ arcsec. 

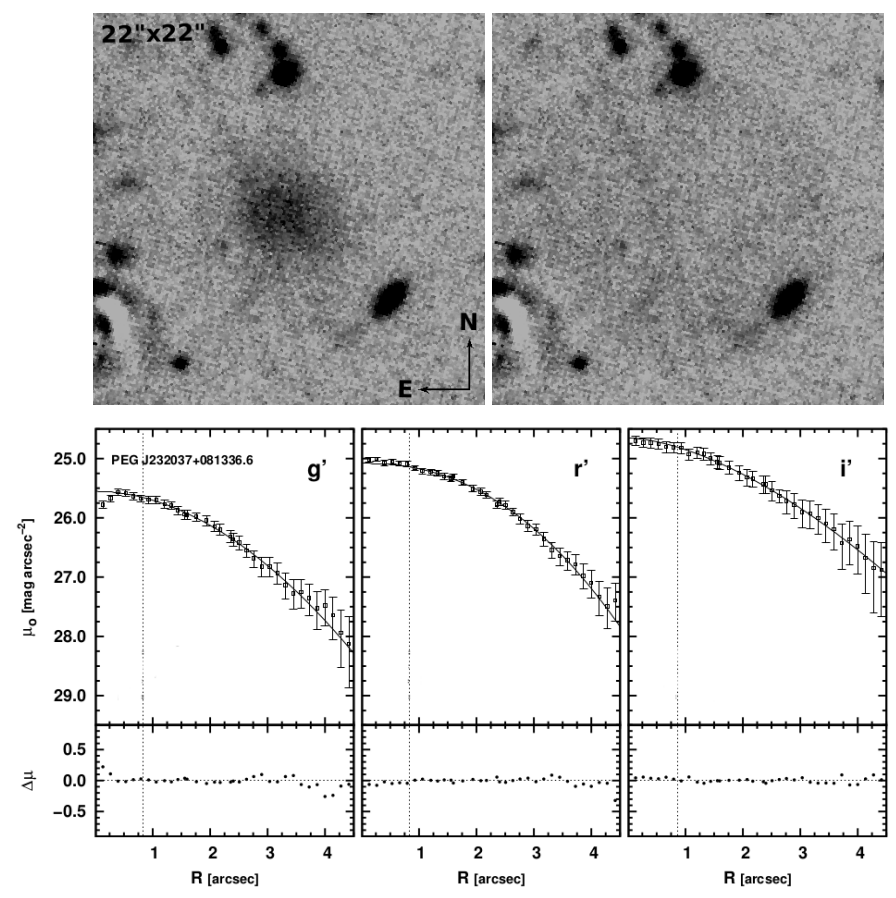

Fig. A.4. As in Fig. 2 but for galaxy PEG J232037+081336.6. In this case, the size of the frames in the upper panels is $22 \times 22$ arcsec.
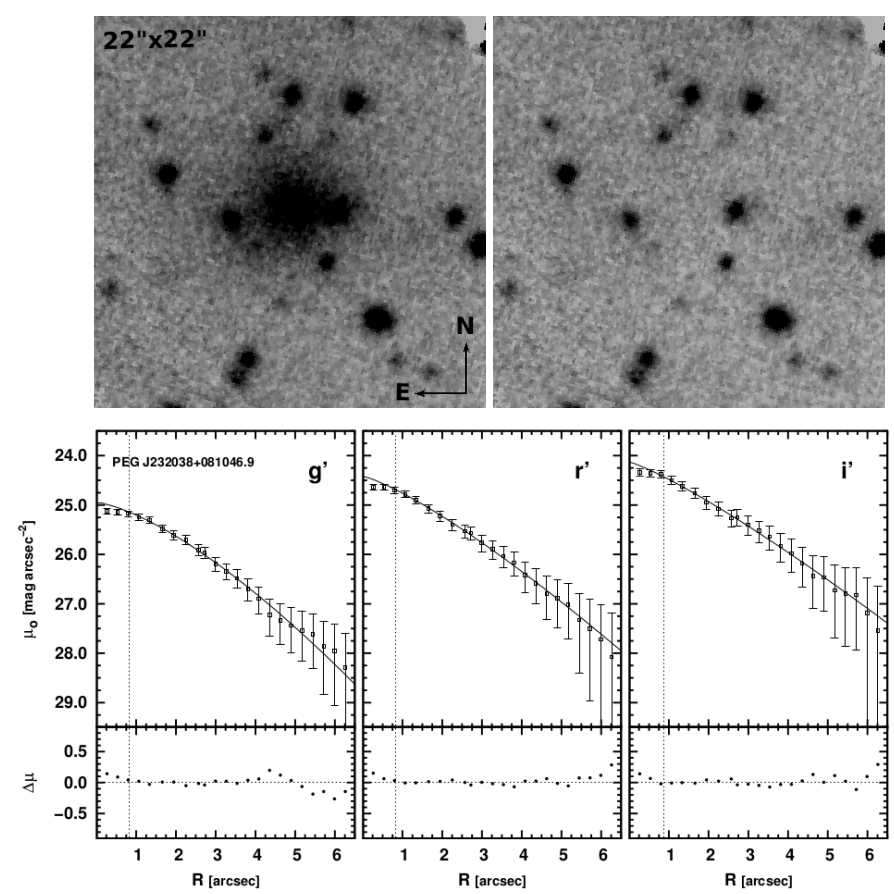

Fig. A.5. As in Fig. 2 but for galaxy PEG J232038+081046.9. In this case, the size of the frames in the upper panels is $22 \times 22$ arcsec.
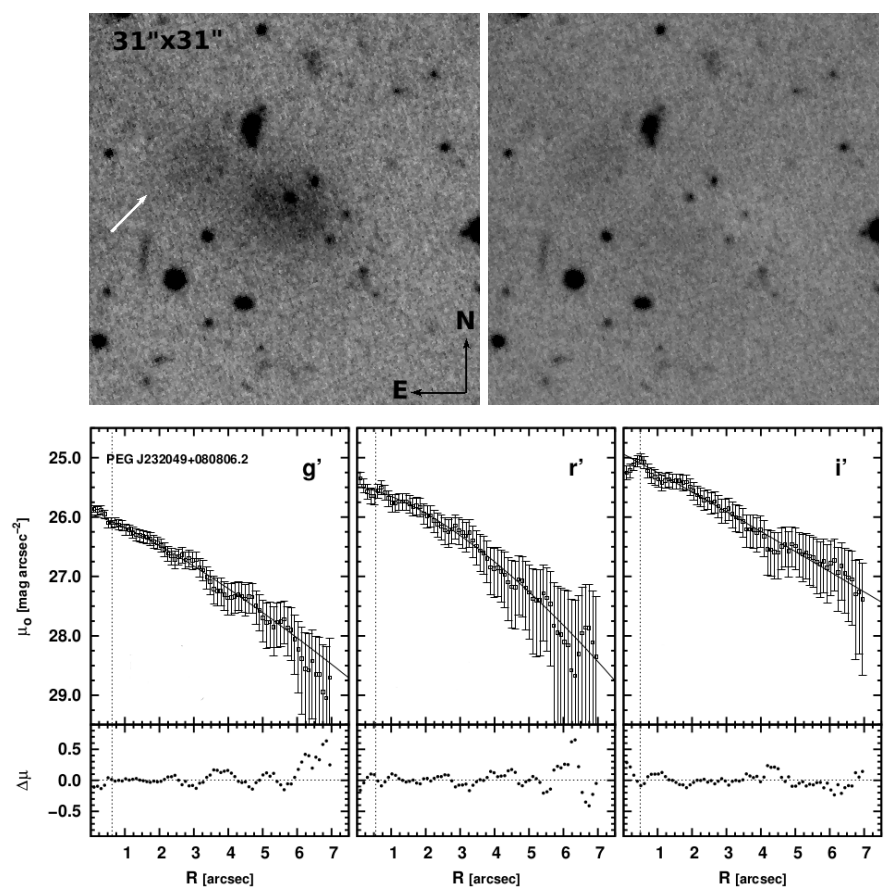

Fig. A.6. As in Fig. 2 but for galaxy PEG J232049+080806.3. In this case, the size of the frames in the upper panels is $31 \times 31 \mathrm{arcsec}$. There seems to be an extremely faint low-surface-brightness object or structure $\left(\mu_{r^{\prime}} \gtrsim 27 \mathrm{mag} \operatorname{arcsec}^{-2}\right)$ to the northwest of the galaxy (white arrow). Quite speculatively, this might be evidence of a tidal origin for this object.
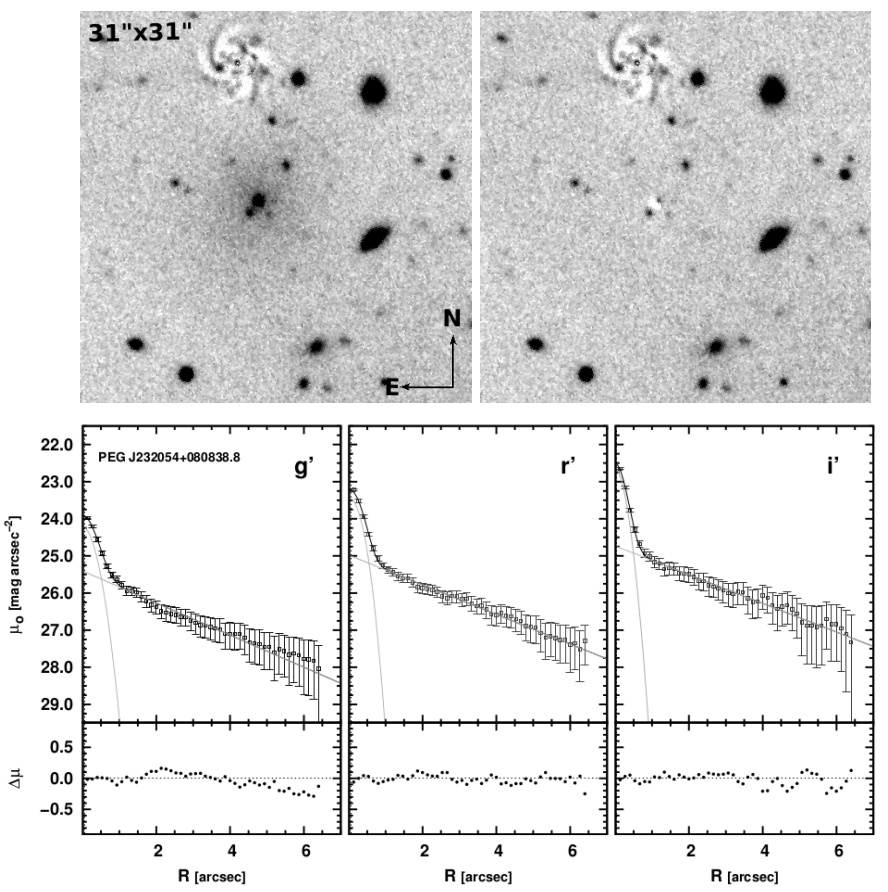

Fig. A.7. As in Fig. 2 but for galaxy PEG J232054+080838.8. In this case, the size of the frames in the upper panels is $31 \times 31 \mathrm{arcsec}$. This object presents a nucleus which is marginally resolved in the $g^{\prime}, r^{\prime}$ and $i^{\prime}$-band images. 